\title{
Labyrinthe
}

41 | 2014-2015

Ici la Dédalie

\section{Ici la Dédalie}

Marc Aymes, Laurent Dubreuil, Laurent Ferri, Grégoire Leménager, Franck Lemonde, Renaud Pasquier et Guillaume Paugam

\section{(2) OpenEdition}

\section{Journals}

Édition électronique

URL : http://journals.openedition.org/labyrinthe/4369

DOI : $10.4000 /$ labyrinthe.4369

ISSN : 1950-6031

Éditeur

Hermann

Édition imprimée

Date de publication : 1 avril 2015

Pagination : 9-87

ISBN : 9782705690717

Référence électronique

Marc Aymes, Laurent Dubreuil, Laurent Ferri, Grégoire Leménager, Franck Lemonde, Renaud Pasquier et Guillaume Paugam, « Ici la Dédalie », Labyrinthe [En ligne], 41 | 2014-2015, mis en ligne le 03 juillet 2015, consulté le 19 avril 2019. URL : http://journals.openedition.org/labyrinthe/4369; DOI : 10.4000/ labyrinthe.4369 


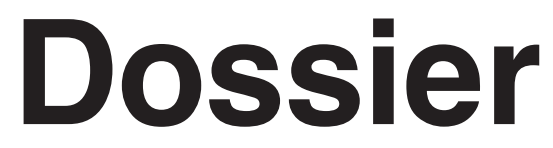

\section{Ici la Dédalie}

par Marc Aymes, Laurent Dubreuil, Laurent Ferri, Grégoire Leménager, Franck Lemonde, Renaud Pasquier et Guillaume Paugam 



\section{L'origine du monde}

C'est une île comme il en existe des centaines, peut-être des milliers d'autres au milieu du Pacifique. Située à un bras de mer de l'île Butaritari, sur son versant méridional, elle ne se distingue par aucune caractéristique particulière. Sauf peut-être qu'elle n'a aucune existence officielle : dans cette zone, les cartes ne mentionnent que les confettis d'atolls encerclant les îles Marshall, dont elle est la proche voisine. Il paraît cependant qu'on peut l'apercevoir sur d'anciennes vues satellites, pour peu que l'on connaisse ses coordonnées précises qui sont, encore à ce jour, maintenues secrètes ${ }^{1}$ (entre $1^{\circ}$ et $4^{\circ}$ de latitude nord, $170^{\circ}$ et $180^{\circ}$ de longitude est, dit-on, ce qui demeure une sacrée surface à scruter). De tous les plus récents relevés topologiques elle a été soigneusement effacée. C'est ici qu'il y a bien des années s'est déroulée l'expérimentation scientifique nommée «Projet Dohrnii ». D'une certaine manière, on peut même dire qu'elle s'y déroule encore aujourd'hui - oubliée de ses commanditaires et en marge de tout protocole scientifique.

L'origine de l'expérience remonte au mitan du siècle dernier. C'était un de ces projets un peu fous, à l'intersection des sciences sociales et de la médecine : évaluer la viabilité d'une communauté humaine placée en totale autarcie, avec en vue les vols spatiaux habités. Nombre de gens ignorent en effet que, parallèlement au programme Apollo qui visait la Lune, et avant même d'en posséder la technologie, on a très tôt envisagé l'hypothèse de voyages extraterrestres de plus longue durée. Mais cela requérait une étude approfondie du facteur humain - aspect qu'avec la mission Apollo 11, qui aura au final duré à peine plus d'une semaine, on pouvait négliger. Un voyage sur Mars suppose, au mieux, six mois de trajet pour l'aller uniquement et, idéalement, un an et demi sur place à attendre la congruence des orbites planétaires favorable au retour - soit

\footnotetext{
1. La plupart des décisions gouvernementales relatives au projet sont disponibles à la bibliothèque du Congrès américain (cote HX810.M67 1999); il n'y est question que du montage administratif et du financement. On se fait une meilleure idée de la nature du projet en le corrélant au dossier de la NASA - la première partie, qui n'a malheureusement pas été numérisée, peut être consultée au Ames Research Center de Moutain View (Californie) en formulant une demande écrite auprès de la $H E O$ (Human Exploration and Operations) Reports Library. Enfin, les archives militaires attenantes au projet (qui contiennent notamment toutes les données médicales et les rapports sociologiques) se trouvent au Redstone Arsenal de Huntsville (Alabama); elles n'ont à ce jour toujours pas été déclassifiées.
} 
au moins trente mois de chasse aux rochers. Avec Jupiter, où l'on pensait alors pouvoir atterrir (on a, bien sûr, été détrompé depuis lors), le trajet aller seul exigerait au moins six années ; pas moins de sept pour Saturne, neuf ans minimum pour Uranus et l'on considère généralement qu'il faudra au mieux compter onze ans pour se rendre sur Neptune - fascinante planète au demeurant mais d'où l'on pourrait également vouloir revenir. Quant aux exoplanètes, les plus attrayantes se situent à au moins une vingtaine d'années... à supposer naturellement un voyage dont la vitesse approchera suffisamment la vitesse de la lumière.

Mais laissons de côté cet aspect pour le moment et considérons ceci : pour les Terriens, en 1960, se rendre sur leur satellite ne constituait qu'un défi technologique. Défi, certes, dont la réalisation fut remarquable - on peut véritablement parler de prouesse ! - mais qui laissait la question humaine totalement de côté. J'en veux pour preuve la rivalité légendaire qu'entretenaient ses deux principaux acteurs, Neil Amstrong et Edwin «Buzz» Aldrin : folklorique, elle n’a jamais mis en péril l’objectif ni le déroulement de l'expédition. Elle servit néanmoins d'avertissement, toute exploration d'une durée et sur une distance plus ambitieuse devait s'appuyer sur un projet de vie à long terme; il faudrait mettre sur pied une communauté viable, c'est-à-dire un groupe d'individus capable non seulement de remplir la somme d'objectifs scientifiques assignée à la mission, mais également de vivre ensemble et de s'auto-suffire, sans interaction physique avec le reste du monde. De l'univers.

L'on pourrait dire aussi que la nature même de l'expérience s'est considérablement modifiée avec la submersion de l'île une vingtaine d'années plus tard et faire remonter à ce point son histoire, mais c'est un aspect sur lequel je ne veux pas trop anticiper. Disons donc ceci : après bien des années d'études préliminaires et de préparation, aux primes heures du 13 janvier 1967 (soit deux semaines à peine avant la catastrophe d'Apollo 1), un groupe de scientifiques, embarqua pour une destination inconnue - cette île même d'Uruk qu'on fit immédiatement disparaître des données topographiques de la région, et dont fut proscrit l'enregistrement dans les relevés cartographiques ultérieurs.

Dans les premiers temps, selon tous les rapports, le projet fut un incontestable succès. Les membres du groupe s'entendaient ensemble à faire fonctionner au mieux leur communauté. Elle comptait quarante-deux membres : vingt-et-un hommes pour vingt-et-une femmes, originaires de pays différents (entendu : du bloc de l'Ouest), recrutés pour leur 


\section{L'origine du monde}

complémentarité. On avait cherché à rassembler une assiette de compétences variées (du docteur à l'ingénieur), des personnes capables tout à la fois d'exécuter des tâches de maintenance électromécanique simple et de réaliser des expériences extrêmement sophistiquées. Il fallait qu'elles soient encore jeunes (il était décisif qu'elles le soient!), aptes à la vie en société restreinte pour un temps indéterminé (disons, pour faire court, qu'on privilégia jardiniers et bricoleurs au détriment des seuls rats de bibliothèque, qu'il fallait être célibataire et si possible orphelin) et, pardessus tout, volontaires. Un tel projet supposait en effet pour les aspirants astronautes de renoncer à participer à une mission spatiale effective, ce qui pouvait faire hésiter. Mais enfin, les militaires ayant été par principe écartés (les problèmes de pilotage et de navigation étant mis de côté), l'échantillon de collaborateurs à disposition se trouvait, par rapport à une mission classique excluant les civils, considérablement étendu. Une dernière sélection se fit au regard de paramètres médicaux (les candidats devaient être en parfaite condition physique) auxquels une consigne officieuse ajouta un critère final, disons «esthétique» : la dernière salve de sélection passée, on demanda aux participantes de désigner, de derrière leur vitre sans tain, les membres masculins qualifiés qu'elles préféreraient pour les accompagner et, inversement, on interrogea les candidats masculins ayant passé la présélection avec succès sur l'attractivité des scientifiques féminines avec lesquelles ils devaient vivre. On croisa ensuite les listes - avoir des personnes se plaisant réciproquement ne pouvait nuire.

Ainsi l'on s'entendit, et l'on se plut. La retraite hors du monde parlait d'instinct à ces jeunes gens avides d'aventures et qui n'avaient encore que peu vécu, la sélection les avait flattés; quant à l'idée de vie en communauté, elle fleurait bon l'époque - bien à rebours de l'esprit militaire animant ceux qui les encadraient, les volontaires pouvaient vivre leur expérience comme la réalisation d'un idéal contre-culturel : on fumait du chanvre; on pratiquait l'amour libre; on discutait cultures, science et spiritualité, vivant à demi-nu au soleil. On refaisait le monde.

Ils avaient raison : il eût été contraire au protocole de la mission de les entraver. Comme il s'agissait de recréer les conditions de vie d'une installation spatiale sur un sol extraterrestre, les membres de l'expédition n'avaient reçu de consigne qu'au plan du matériel : il s'agissait de faire vivre leur campement exclusivement avec ce qui leur avait été fourni au départ. Aussi pouvait-on cultiver, dans des conteneurs appropriés, des plantes dont les graines avaient été apportées, mais non se nourrir des 
baies succulentes qui pullulaient sur l'île, voire de chasser - la consommation de tout autre animal que les lapins et volailles les ayant initialement accompagnés étant strictement prohibée (dans les eaux poissonneuses de Butaritari, la consigne s'avéra particulièrement frustrante, sans compter qu'on eût volontiers fait un sort aux marcassins qui s'habituèrent à visiter le campement endormi; on ne s'étonnera pas que ces règles aient, tôt ou tard, été transgressées). On avait à disposition prodigalité de matériel dernier cri - en quantité inversement proportionnelle aux moyens de communication. Outre la station, les outils de mesure et ceux de production d'énergie, il n'y avait guère qu'un récepteur radio portatif qui pouvait servir à recevoir des nouvelles, ainsi qu'un système de télégraphe pour en donner : c'est-à-dire transmettre des documents écrits, notamment journaux et rapports que, prétendument, il était crucial de tenir avec régularité. Certains de ces documents furent parcourus avec attention; peu, hormis les premiers, furent véritablement lus et quand, bien des années plus tard, il fallut réparer le récepteur dont on s'aperçut enfin qu'il avait grillé, plus personne, à la base militaire de correspondance, n'en maîtrisait la technologie. Ces règles posées, les contacts avec le monde extérieur furent strictement réglementés d'une façon qu'on voulait définitive - aucune interférence extérieure ne devait plus interrompre le protocole; d'ailleurs, une fois la structure de base installée, prolonger l'expérience ne coûtait par définition quasiment rien (soit le salaire des sociologues et quelques faux-frais).

Sur l'île, l'ennemi qu'on avait craint était l'ennui. Des esprits moins brillants se fussent satisfaits de vivre au soleil; le groupe étant d'une intelligence supérieure, il lui fallait trouver de quoi s'occuper. Le rassemblement des intelligences fit merveille, et la description de cette première époque de l'ingénierie îlienne (stimulée par les hallucinogènes ?) aurait tout à fait sa place dans un roman fantastique. Il y eut aussi trois naissances dans les cinq premières années. Or, élever les enfants se faisait, comme tout le reste, en commun. Cela occupa tout ce beau monde un bon moment - sans que l'on sût, au juste, s'il s'agissait d'une conséquence inévitable ou de l'objectif même de la mission; après tout, un voyage sur Neptune impliquait de facto deux générations. On ignorait l'identité des pères, mais c'était égal après tout : on avait recréé sous cloche une société primitive croisée avec une utopie scientifique avancée.

Les îliens s'en amusaient, faute d'avoir autre chose à faire. On simulait des explorations, c'étaient de grandes randonnées dans toute l'île - on 


\section{L'origine du monde}

allait sur les hauteurs, gravir le mont Kish comme s'il se fût agi d'un cratère lunaire, on observait les dauphins, plongeait dans le lagon intérieur, répertoriait toutes les espèces végétales dont on possédait un répertoire circonstancié d'usages (les plantes qu'on pouvait utiliser, tout ce qui était comestible, celles qui faisaient psychotropes); on s'émerveillait de trouver un caméléon, une tortue, un papillon, comme si on avait découvert une preuve de vie martienne. On consignait cela gaiement dans des rapports dont les sociologues, plus d'une fois, suspectèrent l'ironie. Les îliens s'enseignaient réciproquement leurs langues (il y avait au total huit nationalités) et, en plus de l'anglais, on pratiqua bientôt le suédois, l'allemand, l'italien et le français. Elles se fondirent dans un sabir curieux qu'on utilisait entre soi. Tout le monde partageait ses connaissances, on dispensait des enseignements en chacune des spécialités. C'est qu'il y avait là des docteurs en astrophysique, en chimie, en biologie, en mathématiques et en géologie; des ingénieurs en mécanique, en électronique, en robotique et en transmission, un philosophe - sans compter les quatre membres de l'équipe médicale : deux médecins et autant de psychologues (mais pas de poète). Beaucoup à savoir, beaucoup à explorer. On échangeait d'abord à titre de curiosité, pour se connaître, mais aussi on profita de la science des autres pour développer la sienne propre; de sorte que les expériences que, dans un premier temps, on réalisait surtout pour se distraire se complexifièrent. Avant même le tournant de la submersion, la coopération étroite, l'assiduité au travail et l'usage de drogues récréatives avait produit de remarquables résultats - de ceux qu'on aurait bien peine à croire s'il n'en subsistait de nombreuses preuves.

Dans cette première phase, l'expérience dura au total dix ans. Elle ne fut suivie avec rigueur que les cinq premières années. Sur «Terre», le succès des missions Apollo avait politiquement détourné l'attention; la Lune était conquise, on en oublia les échéances de plus long terme dont l'intérêt passait à l'arrière-plan. Et puis, on n'était pas près de posséder la technologie pour ces voyages au long cours qu'on avait imaginés. Bref, il y avait beaucoup plus urgent. L'encadrement militaire, pour sa part, ne se soucia plus que de loin en loin de ces hippies dont ils avaient administrativement la charge mais qu'ils ne voulaient pas comprendre et ne pouvaient commander - sans compter qu'ils ne voyaient pas l'application pratique du projet : ce n'est de toutes façons pas ainsi qu'ils concevaient une expédition en terre inconnue. Quant à l'observation sociologique, elle apparaissait surtout rébarbative. Peut-être l'exploration avait-elle 
été trop bien conçue? On s'était attendu à devoir diagnostiquer des problèmes, élaborer des modèles herméneutiques, proposer des solutions à mettre en œuvre à distance. Or, mise à part la défection de Tobias ${ }^{2}$, le psychologue allemand, il ne se passait rien de bien captivant sur l'île. Et puis, l'expérience était confidentielle - autrement dit : il n'y avait pas de bénéfice à en retirer auprès des scientifiques de la société civile. Ainsi les mandats passèrent, le personnel changea au rythme des élections, on se transmit les secrets-défense dans l'ordre de leur priorité supposée, en renvoyant, pour les détails des dossiers les moins brûlants, aux épais rapports que les nouveaux responsables se promettaient toujours de lire, pieuse résolution que leur diligence s'émoussant ne permettait pas tout à fait de tenir. C'est ce qui arriva au projet Dohrnii : l'intérêt faiblit et, pendant une décennie, on ne se soucia que peu des exilés - pour ce qu'on en savait de ce côté du Pacifique, le projet ne subsistait qu'à l'état léthargique. Du côté des îliens, le flottement se fit sentir. Au bout de quelques années on se mit discrètement à cultiver les plantes indigènes, puis on chassa, on pêcha, on colonisa plus d'espace : avant la fin de la décennie on s'était approprié ce qui restait de l'île (qui, dès le départ, n'était pas bien grande). On vécut donc ainsi, heureux robinsons ayant créé leur modèle de société, fût-elle vouée à une disparition prochaine. Car celle-ci s'approchait.

Conformément au plan, les contacts avec la base avaient été rien moins que sporadiques. Les grossesses avaient pu se dérouler sans suivi extérieur, on n'avait pas eu à recourir à l'assistance médicale jusqu'à ce que Tanysha, la chimiste australienne, ingère de méchants champignons : mais les médecins embarqués parvinrent in extremis à faire retomber sa fièvre (qui sait ce que les autorités auraient répondu... et quand?); quant à l'épisode dépressif de Tobias, il s'était soldé de lui-même par son départ abrupt pour les îles Marshall. Les «naufragés » eurent cependant un impérieux besoin d'assistance. Trois ans à peine après leur arrivée, il semblait déjà aux îliens que leur territoire s'était un rien restreint; à force de relevés topographiques de plus en plus précis, on documenta la chose. Les données ne mentaient pas : l'île rétrécissait année après année.

De ce fait, les habitants n'avaient pas l'explication - l'eau montaitelle ou était-ce la terre qui s'enfonçait? Il leur manquait une boussole d'inclinaison pour conclure à un affaissement de la surface immergée, de

2. Tous les prénoms ont été changés (NDLR). 


\section{L'origine du monde}

l'autre côté, on ne parlait pas encore de réchauffement climatique (ce qui apparaît maintenant comme la plus vraisemblable cause de cet étrange phénomène). Toujours est-il qu'en a peine dix ans, l'île avait perdu presque la moitié de sa surface habitable qui se trouvait au-dessous du niveau de la mer. Les «naufragés» (qui, pour le coup, l'étaient vraiment) s'étaient installés sur le flanc du mont Kish; monticule d'une cinquantaine de mètres de hauteur seulement qui leur offrait un refuge bienvenu. Mais le reste de la surface jadis si hospitalière s'était vu réduit à une poignée de confettis.

Le 25 mars 1977, Gwen Whitman, secrétaire de division à la DARPA (Defense Advanced Research Projects Agency), après plus de six mois d'atermoiements, et bien de la perplexité de se retrouver ainsi «en charge» d'une opération que ses deux prédécesseurs directs avaient négligée, choisit l'option qui demandait le moins de décision de sa part - c'est-à-dire celle qui engageait le moins sa responsabilité, lui demandait le moins de réflexion, et exposait le programme le moins - et accéda à la demande des îliens, déterminés à poursuivre l'expérience dans des conditions plus favorables, de procéder à leur évacuation pour l'île habitable la plus proche : il leur envoya le vaisseau de transport ADFL-23 $3^{3}$ qui réinstalla la mission sur Teirio, à plusieurs miles nautiques de là. Il ne lui appartint pas de décider de la suite, et c'est de leur propre initiative que les îliens, moins de deux ans plus tard, s'installèrent sur l'archipel de Tawara (que l'on peut traduire par : «Monde» ou, autrement, «Dédalie»).

Un mot sur cette décision : les scientifiques ne se firent jamais à la longiligne Teirio - fruste et peu aisée à domestiquer. Exposition au vent, force des courants, rareté de la végétation et manque de ressources endémiques... les défis existentiels y étaient nombreux pour une communauté qui n'y retrouvait aucun des avantages naturels qui avaient rendu Uruk si propice à son épanouissement. Il s'agissait autant de confort que de motifs scientifiques : il n'est plus de temps pour la science dès lors que, jour après jour, se posent sans cesse des questions de simple survie. Mais c'est cette fois en dehors de tout protocole que les îliens se transposèrent sur Dédalie. Sur le continent, Whitman avait été débarqué dans la foulée de l'accession des Démocrates au pouvoir et le poste qu'il

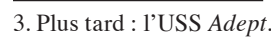


occupait au sein de la DARPA ne fut pas reconduit tel quel ${ }^{4}$. Faute de réponse immédiate à leur demande, les scientifiques profitèrent à ce qu'il semble de la vacance de leur administration de tutelle pour opérer une relocalisation par leurs propres moyens (ce que la proximité de Dédalie facilita) et furent totalement établis dès le début 1979. À partir de ce moment, le projet Dohrnii (s'il mérite encore de porter ce nom) changea radicalement de nature. Et pour cause : Dédalie était habitée.

Les Dédaliens accueillirent les scientifiques chaleureusement quoique sans exubérance (il y avait de la place pour tout le monde et on avait toujours de quoi s'employer); ceux-ci trouvèrent en Dédalie un asile agréable et avantageux. Le territoire de Tawara était vaste, l'archipel auto-suffisant - sans compter que, menacée elle-même par la montée du niveau des océans, Dédalie commença à recevoir de substantiels financements dès le début des années 1980 (de la Banque mondiale et du Fonds pour l'environnement mondial notamment). Au diapason du monde extérieur pour ce qui était de la technologie, Dédalie vivait très en retrait de son bruit... et de ses intérêts. Peu de voyageurs s'y étaient aventurés et pour cause : à ce jour, l'île n'est desservie par aucune liaison régulière; le seul moyen de s'y rendre passe par les îles Marshall (que l'on rejoint au prix d'une escale à Honolulu ou Guam), incommodité que son intérêt touristique propre est assez loin de justifier. Dédalie, de fait, est géographiquement loin de tout et appartient comme à une zone grise de notre espace mental : on sait qu'elle existe, peu comment elle subsiste et rarement où la situer. On ignore presque tout de ce qui s'y passe. Le mode de vie n'y est pourtant pas sans intérêt, d'autant plus que, pour ne citer que le trait intéressant le plus directement l'adoption de nos voyageurs, l'essentiel de la vie s'y déroule en communautés. Non que Dédalie en soit restée à quelque stade tribal de développement (il s'agit d'une société tout à fait «moderne», quoi que cela veuille dire), mais les interactions, les échanges économiques et toute la vie civile s'effectuent en dehors de cadres institutionnels et surtout étatiques (pour des raisons que les scientifiques comprirent bientôt, et qui rejoignaient leurs pratique et intérêt). La prospérité relative de l'archipel et l'aménité de son climat

4. Se conformant aux injonctions de l'amendement Mansfield de 1973 limitant le périmètre des recherches en matière de défense, la DARPA se désengagea progressivement des projets à application non directement militaire. Nombre d'entre eux furent repris par la National Science Foundation; à l'heure de boucler ce numéro, il ne m'a pas été possible de déterminer ce qu'il en fût, et à quelle date, du projet Dohrnii. 


\section{L'origine du monde}

laissent à ses habitants le loisir que beaucoup consacrent à la science, la connaissance et, d'une manière générale, l'éducation. Les Dédaliens sont naturellement polyglottes et la langue dédalienne elle-même est un entremêlement de maori et de français - on rappelle que Dédalie, étape répertoriée sur le trajet de Bougainville, fut semi-colonisée dès avant la Polynésie française... et largement délaissée à son profit. Bref, il y avait avec nos scientifiques beaucoup de lignes de convergence que les deux partis ne manquèrent pas d'explorer. 



\section{Note sur la religion de l'alimentation}

Toute nourriture était sacrée. Selon les archivistes et historiens de Dédalie, nulle diète spécifique n'avait accompagné la création de la colonie. Mais, très vite, s'étaient manifestées une attention et une sensibilité extrêmes à l'égard de l'alimentation. Elles avaient ensuite viré au mysticisme. La plupart des Dédaliens étaient végétariens, avec les grandes nuances que nous allons établir plus loin. Il vaut toutefois la peine de mentionner ici quelques groupes minoritaires qui, tout en ayant en commun avec les autres une forme de religiosité alimentaire, avaient produit des théories en faveur de la consommation de viande. La secte la plus puissante numériquement - et la plus impressionnante dans ses réunions publiques - regroupait les Néanderthaliens. Ceux-ci disposaient d'un grand récit mythique, situant la chute morale et culturelle du genre Homo au jour où sapiens avait supplanté son cousin de grande taille. La seule tâche capable de «reconduire la Vérité près la lisière de l'Ur-Urgrund» - pour reprendre leurs formulations - consistait dans la transformation lente et méthodique de l'humain en son ancien congénère. Cela passait entre autres choses par la chasse et la consommation de viande crue, voire le rituel de la frissa, où les Néanderthaliens, nus et excités, dévoraient un ruminant tout vif, pelage et sabots compris. (La fête se finissait parfois à l'hôpital pour cause d'occlusion intestinale.) Comme les lois de Dédalie étaient fort avancées sur la protection animale, ces réunions avaient lieu un dimanche par mois au mieux, dans l'une des grandes réserves. Les Néanderthaliens recevaient alors autorisation pour leurs élucubrations préhistoriennes, sous le titre officiel de Régulateurs patentés de la biodiversité génétique protégée. Ils accomplissaient une mission d'éradication partielle jugée nécessaire, et on fermait les yeux sur leurs méthodes, en attendant l'introduction prochaine des robots régulateurs que promettait depuis soixante ans l'Institut de DedalTech. De toute façon, peu de Néanderthaliens avaient l'expérience suffisante pour attraper le gibier à main nue, et ces parties dominicales se résumaient donc pour de nombreux officiants à une séance collective de broutage de pré.

Les autres viandards de Dédalie, qui professaient une foi dans la valeur du carné sans pour autant ratifier les superstitions néanderthaliennes, se nourrissaient de muscles artificiellement produits dans des usines laboratoires assermentées. Le repas commençait souvent par une action 


\section{Labyrinthe $n^{\circ} 41$}

de grâce au premier animal, celui qui avait été autrefois sacrifié afin de fournir le paquet de cellules devant être ultérieurement utilisées dans la culture artificielle d'organes comestibles. Une confrérie particulière, les Compagnons du Lion, entretenait une prédilection pour les abats. Parmi ses dictons oraculaires, on trouvait deux promesses assez ridicules et peu assurées, comme qui vit d'abats voit de haut (soit la clairvoyance due au pied de cochon frit) et trip by tripe (horrible jeu de mots sur l'extase hallucinatoire peut-être consécutive à l'absorption massive de gras-double et autre bonnet). Il fallait voir les Compagnons se presser aux magasins de laboratoires et réclamer leurs joyeuses, se pourléchant à l'idée de manger un peu de cette joie parée, spongieuse et douce.

Le troisième groupe omnivore dont il soit utile de parler dans cette note relevait du bifrigisme, qui réclamait à ses adeptes de posséder, pour la plus grande gloire du Divin, deux réfrigérateurs distincts. Dans le premier frigo ne se conservaient que les aliments dont le nom avait pour initiale une lettre allant de $A$ à $M$; dans le second se trouvait logiquement le reste de l'alphabet. Des règles complexes gouvernaient la cuisine, et il était sacrilège de mélanger des nourritures provenant des deux réfrigérateurs. À suivre les bifrigistes, le Divin avait commandé Tu ne cuiras pas le $n$ dans l'e de son $r$, et donc interdit la confusion onomastique. Bien sûr, certaines questions d'interprétation - précisément littérales - demeuraient : selon la langue retenue, la répartition n'était pas identique (or Dédalie était après tout une sorte de seconde Babel), et, à l'intérieur d'un même idiome, des doutes, subsistaient sur l'appellation. La diversité des débats philologiques ainsi occasionnés ${ }^{1}$ expliquait l'attrait du bifrigisme chez les intellectuels omnivores, qui avaient parfois plus de mal à goûter l'aspect concret de la célébration néanderthalienne, ou pouvaient rechigner à se faire monomanes du rognon.

Nous pourrions maintenant évoquer les insectivores ou les herpétophages. Par souci de concision, nous n'entrerons pas dans ces détails et nous tournerons vers la majorité végétarienne. Celle-ci n'était unifiée que par son refus de la chair, fût-il un résultat de l'industrie biochimique. Pour le reste, on observait des lignes de fracture très puissantes, correspondant chaque fois à une irréductible doctrine mystique. On trouvait ainsi les acharnés partisans des noix et cacahuètes, qui certifiaient que leur

1. Par exemple : la Coquille Saint-Jacques est-elle un $C$ ou un $S$, voire un $J$ ? le Radis noir est-il un Daikkon? etc. 
régime les mettait en contact avec le Génie Créateur et Commercial de l'Univers, qui tenait pour eux le rôle de démiurge. À force de pistaches et de cajous, certains pratiquants connaissaient de sérieux dérèglements pancréatiques : s'ils étaient du coup conduits à la mort, on estimait que le GCCU les avait rappelés à lui, et qu'ils fructifiaient désormais ensemble, en un monde meilleur encore que Dédalie.

La séparation végétarienne la plus notable passait entre cuitistes et crudistes. Ces derniers bannissaient tout usage de la chaleur pour transformer les aliments, moyennant, dans leurs rangs, un premier désaccord sur la question du séchage au soleil, procédé vu par les uns comme une sanctification du Sain Manger par le Saint Astre et, par les autres, comme une hypocrite carbonisation de la virginale fraîcheur de la Vie. La distinction entre fruitistes et légumistes était sans doute de plus grave conséquence philosophique, et il convient de rappeler qu'elle affectait aussi bien cuitistes que crudistes. (On pouvait - n'est-ce pas ? - se convertir du fruito-légumo-végano-cuitisme au légumo-crudisme exclusif.) Le grand penseur Pamphilex avait un jour publié un libelle contre les barbares sourds à la plainte du chou cabus pleurant d'être arraché du sol qui le porte. «Si le chou a mal à sa racine, serez-vous assez mauvais pour le dévorer? Mangeriez-vous votre propre mère de la sorte décapitée?», demandait Pamphilex ${ }^{2}$. Cet ouvrage avait connu un succès considérable, et provoqué des expériences scientifiques. Elles établirent sans aucune ambiguïté qu'un légume coupé, tranché, déterré crissait de manière imperceptible à l'oreille humaine mais enregistrable par de fins instruments. Pis, ces sons émis entraînaient des réactions de stress sur les plantes environnantes. Cela confirma que ces bruits étaient des cris de douleur. Comme les résultats pour les fruits n'étaient pas aussi concluants, c'est-à-dire qu'une pomme semblait ne point se plaindre d'être cueillie, les légumistes (y compris les fruito-légumistes) étaient généralement considérés par Pamphilex et ses amis fruitistes comme de véritables ordures, plaçant la malice de l'homme au-dessus de la douleur du Vivant.

D'aucuns cependant étaient entrés dans la tofuphagie, car rien ne prouvait non plus que bananes ou tomates ne fussent pas rendues muettes de douleur. Incertains de la souffrance qu'ils causeraient au monde sinon, les tofuphages ne se nourrissaient plus que de succédanés usinés du soja,

2. Ce à quoi les Néo-pyrrhoniens avaient rétorqué «mais oui, faisons un steak de nos parents », et ainsi de suite. Une loi expresse fit peu après dissoudre ce groupuscule. 


\section{Labyrinthe $n^{\circ} 41$}

leur bénédicité au «premier plant sacrifié» rappelant singulièrement (et paradoxalement) les grâces des viandards. Puisque l'approfondissement de l'intégrisme est toujours possible, une fraction avait déclaré l'égalité de toute vie dans la douleur, et, sur la base d'expériences pourtant contestées sur les larmes de la figue, avait prôné l'abstinence totale. Par défaut d'alimentation, ces partisans étaient morts assez rapidement. Ceux qui, avant de succomber, avaient connu un épisode de coma végétatif, passaient pour les plus saints; on leur érigeait de petits temples en forme de champignons. Hormis les Néanderthaliens, trop rarement touchés par le sentiment d'admiration, les autres Dédaliens, de toute obédience ou régime, gardaient au fond d'eux-mêmes un fort respect pour ces ultimes martyrs de la cause végétale, qui avaient su pénétrer le saint du saint de la nourriture et s'étaient mystiquement transformés en légumes par solidarité avec leur prochain. 


\section{Fragment d'une tablette retrouvée dans les Hautes Terres}

Traduit du dédalien par la rédaction ${ }^{1}$

- C'est ici, n'est-ce pas?

L'être a surgi. Il devait être derrière le rocher que vous voyez là. Depuis quand, nul ne sait.

Il parle notre langue, quoiqu'avec un fort accent. À l'oreille, je ne saurais dire d'où il vient. (Aussi, mes concitoyens et moi-même n'avons des langues d'ailleurs qu'une culture fort livresque.) Ce qui est sûr c'est qu'il n'est pas d'ici. Il semble étourdi, comme quelqu'un qui se lève d'un long voyage un peu comateux. Lui-même se demande manifestement en quelle terre incertaine il est parvenu.

- C'est ici, n'est-ce pas?

Ce sont ses premiers mots. Longtemps il s'en tient à répéter la question, à lente et distincte voix. Est-ce la seule phrase qu'il sache dire? Ou craint-il de ne pas avoir été compris? À en juger par ses paroles, il n'exclut pas de s'être trompé d'adresse. Mais comme il n'a pas dit où il espère être arrivé, personne ne s'est risqué à lui donner tort.

- C'est ici le pays d'Utopie?

Nous y voilà. Il l'a dit lentement et distinctement, mais il a fallu lui demander de répéter, car avec sa curieuse diction personne n'est sûr d'avoir bien entendu. Le pays... de quoi, dites-vous ? Du taux pi ?

- Utopie.

Comme je le disais, la phonétique n'est pas notre fort. On le prie de nous l'écrire. Il trace au sol une suite de six lettres de l'alphabet que l'on dit latin : U.T.O.P.I.E. Puis, à la manière d'un instituteur :

- U.T.O.P.I.E : utopie.

On dut le décevoir :

1. L'ordre adopté pour la présentation des différents fragments dans le présent dossier se fie à la chronologie établie par datation suivant le protocole WayBack. 


\section{Labyrinthe $n^{\circ} 41$}

- Vous n'y êtes pas du tout : ici c'est la Dédalie. Pour ce pays d'euh... d'Utopie que vous recherchez, nous craignons de ne pouvoir vous renseigner.

Non que ce toponyme nous fût totalement exotique. Tenant de nos ancêtres le goût des langues, nous reconnûmes sans peine qu'il devait venir du grec, langue prestigieuse s'il en fut. C'était un jeu d'enfant, il faut dire : $ө o ́ \pi o \varsigma, ~ « l e ~ l i e u » ; o v$, particule privative. Utopie signifiait donc...

— Un «non-lieu»! Voilà ce que vous prétendez chercher? Dites voir, petit plaisantin, pour qui nous prenez-vous?

Nous tournons les talons. Il s'éclipse comme il est venu. 


\section{Éducation, piège à cons}

Une chose qui n'a pas laissé de m'étonner est la passion des jeunes Dédaliens pour les jeux d'esprit et notamment de lettres, et ce dès le plus jeune âge. Demandez à un garçonnet ce qu'il voudrait faire quand il sera grand, invariablement il vous répondra «écrivain » ou «philosophe». Les fillettes prétendant toutes devenir «essayiste » ou «femme de lettres », si les carrières devaient s'arrêter au vœu de la prime enfance il n'y aurait bientôt dans toute la Dédalie ni aviateur, ni vétérinaire, ni pompier. Il m'a fallu bien du temps pour m'acclimater à cette étrange manie, et il n'est guère de peine que je me sois épargnée pour comprendre l'origine d'une telle fascination. On trouvera ici consigné le rapport exact de mes observations sur cet aspect pittoresque de la culture dédalienne.

L'éducation littéraire commence très tôt. Je ne sais trop comment les jeunes Dédaliens apprennent à lire, ceux que j’ai interrogés n'en avaient pas gardé le souvenir; il ne se souvenaient que de leurs premières lectures et de l'effet qu'elles produisirent sur eux : c'est le temps d'où ils dataient sans interruption la conscience d'eux-mêmes. Très jeunes, ils semblent déjà pénétrés de culture livresque, paraissant animés de passions artificiellement ressenties, pétris de récits compassés, enflammés à la simple évocation des mythes de civilisations disparues. Pour moi, je dirais qu'ils apprennent spontanément grâce aux mots croisés qui font la une des journaux : non que cela paraisse vraisemblable, mais cela expliquerait bien des choses (au premier chef que, dans leur manière de considérer la chose littéraire, il leur en reste toujours quelque chose d'un peu alambiqué).

D'une manière générale, ils ont notamment une fascination remarquable pour les jeux de mots et tout procédé d'écriture cryptique. Dès l'âge de sept ans, les écoliers se mettent à faire des vers au point qu'il n'est pas rare de voir les élèves de neuvième solder leurs différends aux bouts-rimés. Les écoliers les plus admirés se distinguent par leur habileté aux hexalipogrammes palindromiques (quand toutes les voyelles ont été proscrites) qui, aux oreilles non averties, semblent des grognements. Plus admirable encore, dans une famille de ma connaissance, les enfants s'étaient défiés de ne jamais employer deux fois le même mot dans la même semaine. Cela faisait deux mois qu'ils s'y tenaient, remarquablement sages, voire mutiques - qualité bien trop rare à cet âge. Dans les cours de récréation, les fleurs de rhétorique fusent ainsi que les invectives contrapétistes 
(équivalent grosso-modo à : «votre père a l'air mutin»; «tes colliers font chic»; «il fait chaud et beau», etc.) et les portes des toilettes, propices aux dégradations, sont couvertes de slogans abscons : «L'Existentialisme est un humanisme»; «Les Non-dupes errent»; «Bosna!», pour n'en citer que quelques-uns.

De manière plus générale, la cloche à peine sonnée les lycéens n'ont qu'une hâte : pouvoir rentrer à la maison se plonger dans leur lecture. Les auteurs français, notamment, sont très prisés - la littérature vernaculaire, particulièrement imbitable, ne se laisse pas si facilement aborder. Ils y passent de longues heures, comme si rien d'autre n'existait. On peut constater qu'ils connaissent extrêmement bien leurs classiques, dont le canon est nettement plus étendu que chez nous. Ainsi, on préfère Xénophon à Platon, on dévore Louis Racine et Jean-Baptiste Rousseau, on ne se contente pas de ce qu'en dit Plutarque : pour briller en société, il faut avoir lu Fabricius. Les auteurs les plus anciens ont le plus de faveur, les plus obscurs paraissent les plus prestigieux - chez les écrivains Mallarmé, Aloysius Bertrand ou bien Raymond Roussel; Héraclite, Hamann ou Benjamin pour la philosophie (laquelle ils commencent à étudier dès l'âge de six ans). Au collège, chacun a déjà son auteur favori dont il dévore le corpus entier - tel Pythagore, Protagoras, Daphnis d'Hylè ou encore Phrynichos - et entendre ces petites têtes blondes (ou rousses, ou crépues, etc., à vrai dire cela n'a aucune espèce d'importance) raisonner sur des sujets réputés ardus n'a jamais laissé de m’impressionner.

Jusqu'à un âge avancé cependant, les discussions gardent un je ne sais quoi d'oblique : n'ayant pas la vue synthétique d'un thème particulier mais une connaissance orientée par l'auteur dont ils sont friands, le savoir des jeunes Dédaliens reste longtemps partiel; sans compter qu'ils se montrent assez facilement dogmatiques, n'ayant pas encore de contrepoint à cette perspective qu'ils ont épousée en totalité. Ainsi, je me rappelle une conversation assez poussée sur la fonction de l'imagination chez Tertullien avec une demoiselle de onze ans qui m'avait épatée... je réalisais bientôt qu'elle n'en savait rien de plus que ce qu'en avait dit Malebranche! Enfin, il m'a semblé que cette prédilection pour les auteurs anciens pouvait rendre la société dans son ensemble assez conservatrice - à tout le moins quelque peu frileuse envers la nouveauté. Comment se fait-il qu'il soit si difficile de se procurer du Amélie Nothomb ou François Bégaudeau en librairie alors que, sur les rayonnages, les auteurs les plus abscons s'amoncellent? D'ailleurs, ils semblent ne pas même savoir qui 
est Michel Onfray - ce qui est un comble pour qui se pique d'érudition ! Celle-ci semble donc, en dernière analyse, toute relative.

Comme ces occupations les détournent de leurs obligations scolaires, les parents dédaliens s'en navrent et prétendent qu'il faut voir dans cette prédilection pour la culture lettrée l'influence néfaste de la télévision (qu'eux-mêmes ne se privent pourtant pas de regarder).

C'est qu'aux heures de grande écoute, les émissions les plus populaires rivalisent d'inventivité pour proposer qui une exégèse du onzième sermon contre les hérétiques d'Augustin, qui une analyse des formes du jugement cartésien, qui la retransmission en direct d'un curetage des condylomes. Soir après soir, ce sont ainsi petits et grands qui restent comme hypnotisés devant leur téléviseur, négligeant le reste de leur vie sociale et domestique. Dès la fin de l'après-midi, les rues des villages se vident, les magasins se ferment, les téléphones ne sonnent plus. Les cœurs, presque, s'arrêtent de battre : chacun est rivé devant le sempiternel petit écran, au risque de veiller jusqu'à des heures très tardives.

Les humanités rassemblent le plus gros des audiences, mais aucune des sciences et des techniques n'est négligée pour peu que le sujet soit suffisamment pointu et son traitement rigoureux; aussi m'a-t-il semblé que le savoir se diffusait par agrégats : somme de connaissances accumulées sur un domaine certes restreint mais exploré exhaustivement. Et le lendemain chacun commentera avec passion les programmes de la veille, démontrant sa compréhension des conceptions les plus abstraites, ayant saisi dans toutes ses nervures le raisonnement scientifique finement articulé, pouvant disserter sans plus de peine sur l'histoire de la Mésopotamie antique ou du deuxième principe de la thermodynamique qu'à propos de la vie sexuelle des platyhelminthes ou les flatuosités des drosophiles.

Reposant sur une formule somme toute basique (des gens ordinaires, moins fréquemment des personnalités, dont les réactions non scénarisées sont captées au jour le jour), le filon des programmes de télé-réalité a eu longtemps le vent en poupe. La plus connue du genre (Fahrenheit story) proposait ainsi d'observer un groupe de personnes enfermées dans un appartement devant se réciter des pages voire des chapitres entiers de livres préalablement appris par cœur. Elle a fasciné une génération entière avant de finir en miettes, supplantée par le suspense d'Eschylor : répartis en deux équipes, des comédiens installés sur une île déserte doivent coopérer pour monter en quarante jours la représentation de l'Orestie 
la plus convaincante. Les perdants sont tellement raillés qu'ils doivent se cacher, finissent par s'exiler, ou font du cinéma. On peut également suivre les émouvantes aventures des derniers copistes qui, malgré leurs rudes conditions d'existence, tentent de rencontrer l'âme sœur - et il faut les voir, ces bougres incongrus et un peu patauds, tenter de toucher les demoiselles par leur belle main...

Les séries pour leur part se sont montrées de tout temps une valeur sûre. Il en existe de plusieurs types, mais il s'agit en général de reconstituer le parcours de personnages du passé hauts en couleur dont elles soulignent l'influence sur le cours de l'histoire : la servante distraite de Plectrude, qui, dans le final de la cinquième saison, oublie la clef dont Charles Martel usa pour se libérer de sa cellule en 715 !; l'assistant de Melvil Dewey au Columbia College (dont le fameux «Sir, this is a mess...» fut de tant de conséquences) qui, à force de patience et de mutations administratives, finira documentaliste à la bibliothèque municipale de Cincinnati; la gouvernante salace des parents Freud. Les décors sont abstraits voire minimalistes, il s'agit le plus souvent de dialogues filmés en plan fixe. Enfin, programmes phares des chaînes de télévision privées (et qui, il faut bien le dire, ont fait leur fortune), les documentaires sont délibérément exempts d'effets de mise en scène afin de ne pas distraire la démonstration - l'austérité étant le gage que leur sujet a été soigneusement circonscrit. L'un des plus grands succès de l'histoire de la télévision dédalienne demeure le mémorable «Tergiversations » qui reconstitue en temps réel la réunion de Lillebonne durant lequel Guillaume le Bâtard convainquit les barons du duché de Normandie de renverser Harold du trône d'Angleterre en 1066. Mais comme il ne s'agissait que de la version montée de dix-sept heures (quand l'ensemble des rushes en compte plus de deux cent vingt-cinq), qu'elle comportait une illustration sonore et que les explications en normand avaient été sous-titrées, les puristes ont tendance à la dénigrer.

Naturellement portés à ne considérer de la connaissance que ce qui leur apparaît le plus subtil, les jeunes Dédaliens se désintéressent totalement de notions plus élémentaires que les établissements scolaires ont le plus grand mal à leur enseigner. Ils sont généralement moins ignorants des ressources naturelles de la république des Kiribati que de la situation géographique de Dédalie - dont nombre d'entre eux méconnaissent jusqu'au nom de la capitale. Alors qu'ils peuvent disserter de longues heures sur les enjeux du mémoire de Parmentier de 1779 (qui, 
comme on le sait, fit le sol des victoires napoléoniennes, fut le support de la révolution industrielle et anticipait l'issue de la Première guerre mondiale), la plupart d'entre eux s'avèrent incapables de distinguer une pomme de terre d'une châtaigne. Autre calamité dont j'ai été témoin : s'ils n'ont aucun mal à discourir sur les mérites comparés du solfège et de la solmisation, ils semblent complètement incompétents dès lors qu'il s'agit de discerner de la musique dodécaphonique une simple série de bruits. L'éducation aux arts plastiques, surtout, se montre intégralement défectueuse : le commentaire d'une nature morte de Cézanne provoque inévitablement des digressions sur les techniques de tissage du rotin, le règne agité de René d'Anjou (également mort à Aix-en-Provence), ou les dix-huit fruits les plus souvent évoqués dans La Recherche du temps perdu. Rien sur l'équilibre de la composition, l'usage de la couleur, la forme des drapés, les défauts de perspective. Pour Le Couronnement de l'empereur Napoléon et de l'impératrice par David, c'est encore pire : le sceptre papal évoquant de manière apparemment évidente un outil de bricolage, il ne sera question que de la philosophie de Nietzsche, de L'Homme au marteau de Jean Meckert et, de loin en loin, de la dissuasion nucléaire dans le Pacifique et de l'odeur pestilentielle des fromages corses.

Les résultats aux examens sont calamiteux dont les exigences, pourtant, ne cessent d'augmenter; ces ratés provoquent la consternation des professeurs qui, aigris, démissionnent à une vitesse inquiétante, et la gesticulation des directeurs d'école. Ceux qui restent s'emploient à combler les lacunes d'une éducation fondamentalement défectueuse tout en se plaignant de ressources bien inférieures à ceux d'une télévision omnipotente - quand bien même les responsables promettent sans cesse de nouveaux moyens. Les résultats demeurent à ce jour mitigés; il n'est qu'à évoquer ce recalé de l'examen de fin d'études dont le journal rapporte qu'il a disserté sur les quarante-huit degrés de signification de l'incipit de L'Étranger d'Albert Camus... tout en omettant complètement le sens littéral (le commentaire de l'examinateur, cependant - «Sa mère est MORTE, crétin!! »- ne fut pas totalement excusé).

Une ambitieuse réforme scolaire, censée compenser les penchants des écoliers à l'argutie, fut pompeusement mise en branle. Ainsi le Collège de Dédalie, plus prestigieuse institution académique du pays, a symboliquement créé une chaire de «Culture populaire des années 1980»; il était prévu d'y parler «Nuit de folie», « Conan le Barbare» et Goldorak. C'est-à-dire que tout, encore, devenait prétexte à spécialisation. Voulant 


\section{Labyrinthe $n^{\circ} 41$}

coûte que coûte en percer le secret, la télévision dédalienne a tenté de débaucher à prix d'or les gloires décérébrées de ses homologues du vieux monde - des animateurs français, particulièrement, auraient été âprement sollicités. Sans succès à ce jour. À défaut, elle a tenté d'imposer à son public des jeux d'argent que personne n'a regardé, de la chanson de variété, des micro-trottoirs d'une platitude remarquable qui n'a pourtant jamais provoqué la fascination escomptée : à mon départ, il n'était encore question que du programme de la veille («Les vicissitudes de la virgule flaubertienne dans les études préparatoires aux Mémoires d'un fou : une approche génétique»).

En désespoir de cause, on parle désormais d'introduire la publicité. 


\section{Utopie}

Fragment d'une tablette retrouvée dans les Hautes Terres.

Traduit du dédalien par la rédaction ${ }^{1}$

C'est une étrange journée

$\mathrm{Ni}$ coloris ni formes

J'ai la tête insonore

Qui je suis je l'oublie

Lorsque nous sommes ensemble

Il n'y a pas de raison

Il n'y a pas de sens

Je suis censée ne rien ressentir

Qui je suis je l'oublie

L'oublie.

Gosse fasciste

Utopie, utopie.

Mon chien a besoin d'oreilles neuves

Qu'à qui mieux mieux ses yeux voient

Faites qu'il vive comme je le fais

Ouah ouah ouah.

Gosse fasciste

Utopie, utopie.

J'ai le monde en câblure

Ainsi rien ne m'échappe

J'ai la caboche fortiche

Ils m'ont faite comme ça.

Gosse fasciste

Utopie, utopie.

1. Le texte se présente comme anonyme. À quelques variantes près, il est d'une similitude troublante avec la chanson écrite et composée (en anglais) par Alison Goldfrapp et Will Gregory (2000) (NDT). 



\section{L'entrée dans les Ordres}

La fête est somptueuse, des mets exquis et des nectars délectables circulent dans la riche demeure magnifiquement parée pour recevoir des centaines d'invités qui rivalisent d'élégance. Introduits par nos hôtes, nous contemplons, bouches bées, les splendeurs déployées par les plus riches familles de Dédalie. Nos guides perdus dans la foule joyeuse dès notre arrivée, impossible cependant de comprendre ce que l'on célèbre exactement, l'objet de tant de magnificence et d'enthousiasme. Nous comprenons cependant bien vite que cette fête a une reine : entourée, choyée, célébrée, la jeune fille, vêtue d'une délicate tunique blanche brodée d'or, une coiffe rouge posée sur ses cheveux tressés, virevolte avec grâce d'un groupe à l'autre, applaudie par les uns, saluée respectueusement par les autres, tendrement couvée par les regards humides de ses parents que ne manquent pas de venir féliciter tous les nouveaux arrivants. À force de tendre l'oreille, des bribes de conversations nous parvenons à saisir : il semblerait que la demoiselle, âgée de vingt ans, soit acceptée, au terme d'un processus de sélection extrêmement sévère, au sein d'une communauté d'élite, les valserristes; impossible en revanche, en écoutant tant bien que mal, au milieu des clameurs, les propos enthousiastes tenus ici et là, de démêler s'il s'agit d'une sorte d'ordre religieux, d'académie sportive et militaire ou d'institut intellectuel où l'on se voue à la connaissance et l'érudition.

«Cela tient un peu des trois à la fois », nous dit en souriant une grande et majestueuse femme brune - elle s'excuse de nous avoir interrompus si cavalièrement, mais propose fort gracieusement de répondre à nos interrogations et s'emploie à satisfaire notre curiosité, avec beaucoup de clarté et de précision.

«La jeune Aspasie, en l'honneur de qui nous sommes tous rassemblés ce soir, fait le bonheur de ses parents par son admission, qui plus est avec un rang remarquable, dans l'Ordre des valserristes, le plus prestigieux du pays, celui où sont formés et affiliés les plus célèbres mérétriciennes (et mérétriciens, même si les femmes sont très majoritaires) de toute la Dédalie. C'est, pour une famille, une forme de consécration : d'abord (même si l'entrée n'est qu'un début, et qu'il faut lutter pour se faire une place de choix au sein de l'Ordre) parce qu'être membre annonce avec certitude une situation sociale et financière plus qu'enviable, et la garantie 
d'une vie confortable, sinon aisée; ensuite, parce que, hors de toutes considérations matérielles, le statut de mérétricienne est extrêmement prestigieux en Dédalie. Cela est dû, d'abord, à ce qu'on ne le maîtrise officiellement qu'après de longues et difficiles études, en deux temps, d'abord pour accéder aux grandes académies (les valserristes donc, mais aussi les hiltonistes, mataharistes, et bien d'autres), puis au sein de celles-ci, où la formation est affinée et complétée.

L'enseignement y est de fait extrêmement exigeant et complet, divisé en deux grands parcours complémentaires, l'ars erotica et la scientia sexualis. La première relève avant tout du corps, des mille et une techniques porteuses de plaisir pour les sens : on y apprend donc des rudiments d'arts plastiques, la maîtrise d'un ou plusieurs instruments de musique, la science diverse et subtile des caresses, la connaissance des parfums et de leurs effets, la grâce disciplinée de l'art chorégraphique, le contrôle absolu de chaque organe à la manière des arts martiaux. Il faut être déterminé, souple et courageux pour supporter les terribles entraînements infligés par des maîtres inflexibles.

Les efforts que requiert la scientia sexualis ne sont pas moins considérables, mais se situent sur un autre plan, plus érudit, réflexif et langagier : on y médite les notions mêmes de plaisir, de beauté et de charme, on nourrit ces réflexions d'études philosophiques, historiques et juridiques, en comparant les lieux et les époques, pour y comprendre quels sens on attribue à ces concepts, et quelles places on leur accorde dans l'arrangement social, et puis on pénètre aussi dans les tréfonds de l'esprit humain pour tenter d'y saisir les ressorts du désir, de la séduction, des jouissances.

Mais cette bipartition ne doit pas masquer la profonde convergence entre les deux pôles de cette formation. Ainsi les cours d'Art de plaire, les plus cruciaux, relèvent-ils simultanément de l'ars erotica et de la scientia sexualis : alimentés par les œuvres des plus grands rhéteurs de l'histoire comme des maîtres de la technique théâtrale, ils visent à parfaire l'harmonie du mot et du geste, le savoir de l'élocution, et l'habileté à jouer des plis et replis du discours dans l'infinie diversité des charmes du mouvement.

Vous aurez compris que bien peu se révèlent capables d'aller au bout de cet abondant programme d'étude, et moins encore celles qui gagnent leur place dans les Ordres les plus renommés - car la concurrence est féroce, entre eux et parmi chacun. Les moins douées et courageuses abandonnent donc et se rabattent, presque toujours avec succès, sur 


\section{L'entrée dans les Ordres}

des professions et activités moins prestigieuses - dans la magistrature, l'enseignement, le théâtre, la cuisine et la publicité, entre autres. Les élues des Hauts Ordres, eux, sont enviées et recherchées par toute la société dédalienne. Il nous apparaît évident - à vous aussi sans doute - que la recherche du plaisir, et par-dessus tout du plaisir tiré de corps humains vivants, est un moteur essentiel, - sinon le moteur - de toute activité humaine. Dès lors il est logique qu'une société comme la nôtre, fondée sur la quête de perfection en tous domaines, prenne très au sérieux cette question centrale du plaisir, et encourage la formation de professionnelles en la matière. Ces expertes ès plaisirs peuvent répandre leur science et ses bienfaits dans une société qui y gagne en bien-être, en équilibre, en efficacité et en productivité. Très tôt, nous apprenons à les connaître : le premier contact a lieu à l'adolescence, il est décidé par notre médecin qui, après un rigoureux examen, nous décrète parvenus à maturité physique et psychique; il détermine par de savants calculs le jour et le contexte idéaux, ainsi que les caractéristiques requises de l'initiatrice, ou l'initiateur, (sexe, âge, morphologie, tempérament, etc.); le choix final reste à la famille - plus elle est riche, plus elle recourra à un Ordre élevé dans la hiérarchie sociale, donc forcément plus coûteux, mais le succès de l'expérience sera garantie.

L'initiation n'est cependant qu'une occasion, la première, la plus cérémonielle et sans doute la plus émouvante, de faire appel aux Ordres mérétriciens. Elle est toujours suivie de beaucoup d'autres, très fréquentes. Il est en effet fort mal vu, en Dédalie, d'entretenir un commerce sexuel avec des amateurs, c'est-à-dire des individus dépourvus de tout emblème d'un Ordre, acceptant un rapport gratuit - qui témoignent par là de leur négligence, leur piètre exigence, et, en somme, de leur médiocrité. Cela vaut pour les célibataires - à moins d'un engagement formel avec le ou la partenaire, nous sommes très stricts sur ce point - et a fortiori plus encore pour les couples liés par des vœux éternels, car la fidélité est pour nous une valeur cardinale. En revanche la fréquentation régulière des mérétriciennes agréées est très chaudement recommandée : elle démontre à la fois l'humilité, la volonté d'amélioration personnelle, le souci d'autrui, surtout, et un louable désir de faire partager les connaissances acquises ou ravivées par un talent expert. Qui manque d'assiduité se verra bien vite mal considéré, d'abord par son ou sa partenaire de vie, mais également par ses collègues de travail, et en définitive par toute la communauté dédalienne.» 
Le récit et les explications de la femme en noir nous avaient tous laissé songeurs. L'un d'entre nous, cependant, s'extrayant de sa rêverie, lui demanda si la situation qu'elle avait dépeinte rencontrait l'accord de toute la population ou si des voix contestataires se faisaient entendre. Elle sourit: «Bien évidemment, comme vous l'imaginez, l'unanimité n'est pas pensable sur de tels sujets... Il y a plusieurs débats, interminables et insolubles, plus ou moins pertinents ou intéressants. La critique la plus négligeable est celle des "amateuristes", qui dénoncent le "conformisme hédonique" et l'“académisme charnel" à grand renfort de slogans péremptoires, et, si vous voulez mon avis, très vains. Elle émane en général de jeunes gens impulsifs, qui, l'âge venant, rentrent bien vite dans le rang, si ce n'est quelques rarissimes irréductibles en quantité négligeable; elle peut aussi être le fait d'originaux, d'excentriques, amoureux de la transgression, beaucoup plus discrets que les jeunes rebelles éphémères, et qui, de fait, ne tiennent pas spécialement à faire école, justement parce que la règle leur est nécessaire comme repoussoir, mais surtout parce qu'ils appartiennent souvent aux plus hauts cercles de la Dédalie, et se jouent avec délices de lois dont ils sont, en quelque sorte, les garants et bénéficiaires. Ainsi susurre-t-on que Messaline Valserra, Chancelière supérieure et fondatrice de l'Ordre qui porte son nom, le plus prestigieux, celui qui accueillera donc notre chère Aspasie, n'hésite pas à s'unir occasionnellement incognito et gratuitement à de parfaits inconnus tout ahuris de cette faveur inouïe : se voir comblés pour rien par le corps le plus désiré de toute la Dédalie. Mais ce ne sont là peut-être que racontars, Messaline adore faire parler d'elle, et sait pour cela s'environner d'un halo de rumeurs et de mystère qui étoffe encore sa renommée, et celle de son Ordre. C'est bien cela, d'ailleurs, la seconde critique, beaucoup plus juste, celle-ci : l'insolence élitiste, l'arrogance, même, des Dédaliens issus des Hauts Ordres mérétriciens - la hautaine Messaline incarnant une sorte de paroxysme en la matière. C'est d'autant plus vrai que leur influence s'étend à bien d'autres domaines, grâce aux réseaux tissés aussi bien pendant leur formation que par leur activité professionnelle : toutes les portes leurs sont ouvertes. La Dédalie, pourtant soucieuse d'égalité, tolère les privilèges considérables conférés de facto aux membres des Hauts Ordres. C'est enfin un autre principe d'égalité que bafouent les Ordres, celle qui doit régner entre les sexes, comme c'est le cas en général dans la société dédalienne. Mais dans les Ordres, pour d'obscures raisons socio-historiques, ce sont nous, les femmes, qui dominons très 


\section{L'entrée dans les Ordres}

largement, et qui profitons donc bien plus que les hommes des pouvoirs propres à notre situation sociale. Je le regrette, évidemment, et salue tous les efforts faits pour diminuer ces différences, mais il faut être lucide, cette inégalité ne sera résolue ni facilement, ni rapidement. Mais vous allez devoir m'excuser, je dois vous laisser, j'ai des amis qui arrivent, passez une excellente soirée, ce sera un grand plaisir pour moi de vous recroiser à Néo Cnossos.»

\section{$* * *$}

Nous la regardâmes s'éloigner, avant de nous laisser happer par les festivités splendides de la nuit dédalienne, qui ne nous laisseraient plus tard que des souvenirs brumeux et mutilés. 



\section{Cher François,}

Ouf, enfin cinq minutes pour t'écrire un mot. J'ai cru que je n'y arriverais jamais. Et puis finalement quand on veut... Le voyage s'est bien passé. Je me sens déjà presque chez moi. Comme je suis arrivé dimanche matin, j'avais peur de débarquer dans une ville qui roupille. Je me plantais complètement : les supermarchés, les banques, les magasins de fringue, les boutiques de bricolage, les vendeurs de lunettes, tout est ouvert le dimanche. C'est une journée comme les autres, en fait. Ça ne veut pas dire que tout le monde travaille, il y en a qui prennent leur congé ce jour-là. Mais ça tourne. En gros, chacun a droit à un dimanche par semaine, si j'ai bien compris, mais pas forcément le dimanche. Tu me suis?

Ça semble assez bien fonctionner. On prétend que le chômage n'existe pas, du coup. Je ne sais pas si c'est totalement vrai, mais le fait est qu'on ne voit personne faire la manche dans la rue - ou alors c'est avec une guitare, des ballons à vendre aux gamins, une pancarte pour te proposer de venir faire le ménage chez toi, des trucs comme ça. Une chose est sûre : il y a des gens partout. Quel que soit le jour de la semaine, ceux qui ont pris leur dimanche en profitent pour faire leurs courses, du karaté, se photographier dans la rue, boire des bières, changer de smartphone, et même aller aux putes. Il paraît que c'est un loisir très à la mode ici. On trouve que c'est excellent pour la santé, le moral et la pérennité conjugale. J'hésite un peu à essayer, j'ai encore du mal à oublier Virginie. Je sais, je sais : je suis trop romantique pour comprendre quelque chose à la vie, ça n'a pas changé.

En tout cas, ce qui est bien, c'est que je ne devrais pas avoir de mal à trouver du boulot. Il faut juste que je ne sois pas trop exigeant sur les horaires : quand tu débutes, tu ne choisis pas. Tu prends ce que les autres te laissent, en fonction du moment où ils tiennent à prendre leur «dimanche». Sinon au pire, je pourrai toujours donner des cours de français. Il y a plein d'institutions qui donnent des cours supplémentaires de tout ce qu'on veut, notamment grâce à des systèmes de web conférences payantes assez sophistiquées. C'est aussi à ça que servent les jours de repos, en fait. À apprendre des nouvelles choses : la cuisine marocaine, le tennis de table, le chinois, le trombone à coulisses, le massage thaïlandais, le Javascript, les bases du marketing digital... Pour la plupart, l'idée est de rajouter des lignes sur son $\mathrm{CV}$. De travailler plus pour, un jour, espérer 
gagner plus. Mais il y en a aussi qui font ça par pure curiosité, pour se détendre si tu préfères. La formule à la mode, ici, c'est qu'il faut savoir «s'oxygéner les neurones» de temps en temps.

Évidemment, le français n'intéresse plus grand monde. Les gens sont bien conscients que ça n'a pas d'utilité directe. Et quitte à s'oxygéner la tête, ils préfèrent suivre un tutoriel pour progresser à Battle 7 ou à Candy Crush III. Mais on m'a assuré qu'il reste des snobs qui trouvent très classieux de maîtriser la poésie de Michel Sardou et les aphorismes de Jean-Claude Van Damme en VO. Du coup, ils cherchent des profs pour leurs enfants. Les plus touchés par les cours complémentaires, ce sont eux. Pas sûr qu'ils en aient tous vraiment envie, les pauvres mômes. Jamais une seconde pour s'ennuyer tranquilles. Jamais un dimanche, si tu préfères. Même les parents assez peu friqués les poussent à mort, très tôt. L'idée est simple : c'est pour que leurs gamins soient meilleurs que les autres dans un maximum de domaines.

Cela dit, si on en juge d'après le niveau moyen, ça porte ses fruits. J'ai été accosté hier soir dans un bar par un jeune gars qui avait repéré mon accent. Il s'appelle Georges. Il connaît un nombre de trucs hallucinant sur notre pays : ça allait de la liste de nos 400 fromages à la filmographie complète d'Alain Delon en passant par les pires dessins d'Hara-Kiri et les cours de Foucault sur le libéralisme, dont il m'a récité des pages entières. Il a juste eu l'air un peu interloqué quand je lui ai demandé à quelle heure fermait le bar (j'étais claqué) : «Pourquoi tu veux que ça ferme ?», il m’a répondu. Ici, les nuits aussi sont des journées comme les autres.

Je t'embrasse, la suite bientôt.

Paul 


\section{Rétif, le guide}

Fragment d'une tablette retrouvée dans les Hautes Terres.

Traduit du dédalien par la rédaction

- Nous y sommes, n'est-ce pas?

C'est chaque fois la même chose : à peine réveillés ils ne se tiennent plus d'impatience. Des enfants dans un parc d'attractions.

- Nous y sommes, n'est-ce pas? C'est bien ici l'Utopie? Oh, dites? Dites, M'sieur Rétif, montrez voir! Faites-nous visiter... que nous ne soyons pas venus pour rien!

Rétif, c'est moi. Je leur sers de guide. Depuis le temps, je devrais m'être habitué à leur lubie. Mais non : encore aujourd'hui, je peine à réprimer mon alarme. Rétif, ça je le suis, et comment! Mais il faut bien vivre, alors je prends sur moi. Je fais le tour-opérateur.

Que vont-ils imaginer? Que se racontent-ils à notre propos? Je l'ignore, mais cette histoire d'Utopie fait visiblement partie de leurs classiques. À la moindre occasion c'est le même langage. Et tous de reprendre en chœur.

- Jusqu'à ce que l'on sache pour Dédalie, l'Utopie restait terra incognita. C'était, comme dit l'autre, un non-lieu. L'intuition était là : il fallait que quelque part l'Utopie soit - mais rien ne semblait jamais devoir la confirmer.

Soit dit entre nous, je ne m'explique guère que ces dévoués zélotes soient si pleins d'eux-mêmes. Concédons-le, leur maîtrise de notre langue a de quoi laisser pantois. J'ignore si notre Dédalie est leur Utopie comme ils semblent s'en être persuadés, mais une chose est sûre : leur utopien est du dédalien tout craché. Avec, peut-être, un je ne sais quoi de contrefait dans l'idiome. Mais pourquoi donc prennent-ils si visiblement plaisir à s'écouter parler, à grand renfort d'archaïsmes et de préciosités ? S'estiment-ils uniques détenteurs du seul savoir qui vaille? S'ils y revenaient : de parler savant, nous n'en manquons pas! Ne vous fiez pas au quasi-désert de nos académies : la raison n'est pas que nous en moquions les matières, c'est plutôt que nous les faisons nôtres par d'autres moyens. Là où d'autres passent leur temps aux jeux de toupie, nous compulsons, computons. Foin 


\section{Labyrinthe $n^{\circ} 41$}

de l'avis des pédagogues patentés! À l'âge qu'ils disent «de raison», nous sommes déjà des compendia bipèdes.

Un rapide sondage hexagrammatique nous a donc suffi à le constater : les Utopistes ne sont pas nés d'hier. Bref, ces mufles n'ont rien inventé, et nous savons aussi bien qu'eux ce que leurs histoires d'Utopie veulent dire. Ont-ils eu, de cela aussi, l'«intuition»? Mais pourquoi s'obstinentils à camper leur Utopie chez nous? Assurément, je ne sache pas que quiconque parmi nous s'en réjouisse : tout le monde préférerait qu'ils ne nous aient jamais retrouvés.

Enfin, je veux dire : qu'ils ne nous aient jamais trouvés, bien sûr. 


\section{Relation de l'avènement de Paul $\mathbf{I}^{\text {er }}$ le Bon, de Manon l'Espiègle et d'Alexandre le Placide}

Au commencement, les Dédaliens vécurent heureux et eurent beaucoup d'enfants ${ }^{1}$. Mais la vitalité de la communauté posa bientôt la question de son organisation : il fallait s'assurer que soient équitables les décisions à prendre intéressant le bien commun. Car celles-ci se multipliaient. La distribution des tâches collectives, l'orientation économique, la représentation auprès des entités voisines, la distinction des mérites... de toutes parts des questions s'élevaient qu'il fallait trancher, imposant la tenue d'une assemblée constituante.

La volonté de s'engager dans un projet commun ne faisant pas la communauté de vues, chacun se présenta avec une vision différente de la direction à adopter qu'il estimait naturellement la meilleure pour tous. L'assemblée réunie, les positions semblables émergeant se reconnurent, les intérêts s'agglomérèrent, bientôt se constituèrent des groupes prêts à faire valoir leurs intérêts. Les producteurs et travailleurs de la terre voulurent que primât leur perspective : promettant d'assurer l'autosuffisance alimentaire de Dédalie, il s'agissait pour eux d'obtenir un poids décisionnaire qui reflétât leur importance cruciale pour la communauté; ils savaient ce qui était bon et s'offraient d'orchestrer les travaux à venir, la prospérité étant à portée. Savait-on, par exemple, qu'il suffisait que chacun donnât quotidiennement ne serait-ce que quelques heures de manœuvre pour que les rendements agricoles assurassent définitivement la viabilité de Dédalie?

À elle seule cette suggestion révulsa les négociants qui dénoncèrent le travail forcé, rétorquant que priorité devait être accordée au commerce : en augmentant les échanges avec les pays limitrophes, Dédalie tisserait des liens économiques (et bientôt diplomatiques) réciproques avec les puissances voisines, s'assurant de leur bienveillance et faisant accroître

\footnotetext{
1. Ce récit se fonde sur trois sources : les comptes rendus officiels et articles de presse d'époque pour ce qui est de la période la plus ancienne couverte, le fac-similé des Mémoires de Jacques-Jean Soureau (manuscrit dactylographié, 120 p.) consultable en ligne, et les témoignages d'ethnologie recueillis dans les Proceedings of the 5th Pacific Asia Conference on Language, Cuisine, and Culture, University of Honolulu Press, 1983. Ces documents demeurant très parcellaires, j'ai plus d'une fois été amené à préciser dans le sens de ce qui m'apparaissait le plus vraisemblable. (NdA)
} 


\section{Labyrinthe, $n^{\circ} 41$}

le nombre de devises en circulation sur son territoire, gage de la convertibilité de sa propre monnaie. Cette proposition provoqua l'ire d'un ancien professeur d'économie qui craignit que Dédalie ne se rendît dépendante de l'extérieur. Il usa de mots qui faisaient peur («balance commerciale», « déséquilibre», «dette souveraine», « déficitaire», etc.), mais qui, faute d'avoir été usés depuis beau temps, ne furent pas bien compris. Il fut aisément décrédibilisé par les commerçants : il avait professé l'économie? La belle affaire! C'est qu'il ne l'avait pas pratiquée et ne devait donc pas être entendu. Son avis se trouva donc rapidement marginalisé... mais ses arguments portèrent tout de même : aux ingénieurs qui, le lendemain, réclamaient les moyens de développer la production énergétique, il fut demandé de ne pas recourir aux technologies de l'ancien monde qui eussent rendu Dédalie dépendante et en ressources, et en procédés. Frustrés ils se braquèrent, menaçant à demi-mot de s'opposer à toute motion jusqu'à ce qu'on lavât l'outrage. Enfin ceux qui prétendaient à un office public parlèrent; ils voulaient un emblème afin de se procurer au plus tôt du papier à en-tête. Il y eut un flottement, on ne sut que répondre. Un petit groupe d'indécis se prononça enfin : ils voulaient davantage de bancs; il en fut apporté. Il était tard déjà, la discussion devenant confuse la séance fut levée.

Au deuxième jour et le jour suivant, on ne progressa que dans l'identification des partis. Les groupes se comptèrent, on s'orientait vers un rapport de forces - personne ne paraissait vouloir céder. Il fallait d'urgence frapper la monnaie, mais on ne s'accordait ni sur son poids, ni sur son effigie. On entendit les médecins grommeler qu'ils ne pouvaient se fournir; les transporteurs, qui attendaient des livraisons, s'impatientaient; le personnel d'entretien se lamentait. Les ouvriers avaient apporté des cartes, jouaient, ainsi que du vin. Ils s'égayèrent, on les blâma, fustigeant leur tempérament. Heureusement on ne comptait pas de poètes ! C'est au soir de ce troisième jour que se leva un homme d'âge très avancé, dont la voix chevrotante portait peu mais qui, parlant distinctement et avec résolution, avait réussi à provoquer le silence autour de lui. Le vieux Soureau, ainsi qu'on le nommait, prétendait avoir étudié la loi (ce qui ne pouvait être faux, dès lors qu'on s'en vantait); on l'écouta méfiant : il était sur son terrain. Pour ordonner les discussions, il proposa que les décisions constitutionnelles fussent prises à l'unanimité. À cette heure et faute de mieux, l'idée parut incongrue sans que personne ne trouvât à s'y opposer. De fait, comme la proposition mécontentait tout le 
monde également, elle recueillit d'emblée l'assentiment de chacun. Les commerçants y voyaient une manière de contrer les producteurs, qui souhaitaient s'allier les ingénieurs. Les administrateurs souhaitaient en finir, les enseignants ne croyaient pas qu'elle pût fonctionner. Les artisans s'en foutaient éperdument. La motion fut adoptée à l'unanimité, preuve qu'elle fonctionnait. On se regardait, bouche bée; puis on se sourit, interdit. Les regards demeurèrent un instant incrédules, jusqu'à ce que les applaudissements, timides d'abord, qui montaient de toutes parts, et des rires, rompissent le silence : on tenait une constitution! En un instant, l'euphorie gagna. Les vivats grondèrent, les chapeaux volèrent, on vit une jeune fille jeter sa culotte - quelle fête ! L'amertume se dissipa avec la nervosité, on s'embrassait vivement et se félicitait. Les tensions nées de l'avant-veille tombaient, les rancunes s'évanouirent, tous allèrent se coucher satisfaits du devoir accompli.

Le lendemain cependant, les difficultés jaillirent : qui pour proposer, ratifier et édicter la loi ? À nouveau des camps se formèrent. Au groupe des collectivistes, ceux pour qui il fallait tout décider de concert, s'opposèrent les légitimistes, qui voulaient que l'exercice du gouvernement soit réservé à un exécutif fort. Les uns parlaient «responsabilité», « communauté», « discussion»; les autres «responsabilité», « communauté», « décision». Au rêve d'appropriation collective du destin de la cité répondait le désir de son incarnation par un leader visionnaire. La température, comme le ton, monta; les insultes fusèrent; «fasciste», " anarcho-fédéraliste», « khmer vert», « bureaucrate»; on en oublia le souper. Les plus hardis faillirent en venir aux mains. Au soir, c'est toute la salle qui tonnait; il fallait en finir. La confrontation qui avait duré tout le jour ne put cependant être tranchée : mises aux voix, aucune des deux motions ne recueillit l'unanimité. On se départagerait le lendemain au prix d'un compromis voulurent se rassurer les pragmatiques (conspués à demi-mots par les deux camps).

Au matin du cinquième jour, on n'entendit plus guère les collectivistes encore abasourdis par le renoncement de la veille - quand le peuple de Dédalie réuni en assemblée constituante avait de fait renoncé à son pouvoir de légiférer in persona. De dépit, ils s'étaient auto-dissous. Mais les légitimistes eux-mêmes se trouvaient bien penauds, incapables de persuader au-delà de leur camp du caractère visionnaire de leur perspective et dès lors bien obligés de reconnaître l'échec à faire advenir en leur sein ce leader charismatique dont ils prêchaient la nécessité. Leur ardeur se dissipa. 
Le silence des uns et des autres laissait le champ à l'examen du principe de la représentation. On avait compris la veille qu'il était dangereux de s'en remettre à la vision d'un guide - a fortiori s'il s'avérait absent; les jours précédents avaient montré qu'on ne pouvait décemment mobiliser tout le corps social à seules fins de législation; la délégation du pouvoir à des individus désignés paraissait la seule voie possible.

Encore fallait-il s'entendre sur le mode, la durée et la nature du mandat. La discussion s'avérait prometteuse. Cette fois les bourgeoises avaient préparé des sandwichs; au fond, on faisait circulait le vin de l'avant-veille, bref, tout le monde était prêt. On s'efforça d'en examiner méthodiquement tous les aspects. L'établissement d'un système électoral paraissait fastidieux, la liste des devoirs civiques à établir arbitraire, leur nature contraignante. Comment s'entendre sur les attributions accordées aux membres de la députation? Il fallait évidemment qu'ils aient les mains libres, mais sans qu'ils puissent interférer avec les intérêts particuliers. La durée posait sa part de problèmes épineux : un mandat long donnait aux représentants la liberté d'agir sans craindre les volte-face de l'opinion, mais il les éloignait des nécessités de ce corps social dont il se revendiqueraient l'émanation. Fallait-il alors vérifier le mandat par une réélection? Le terme unique permettait le renouvellement de ses récipiendaires tandis que leur réélection assurait le bénéfice de l'expérience acquise. Il fallait des esprits forts, puisqu'on avait pris en haine les tergiversations. Mais si on se trompait? Le représentant devait donc en savoir suffisamment pour se faire sa propre opinion tout en considérant les avis pour ne pas s'y enferrer. Valait-il mieux pour cela un savant technicien ou un gentilhomme formé aux humanités? Le premier saurait mieux traiter des questions particulières, le second pourrait davantage embrasser la perspective générale. Peut-être faudrait-il tout simplement les associer? Une collaboration posait la question de l'étendue de leurs compétences. On se demandait lesquelles, au juste, étaient nécessaires. Et puis n'y aurait-il pas des conseillers? Mais leur nombre nous grèverait d'impôts! D'ailleurs comment rétribuer la députation? S’il paraissait naturel de dédommager le temps soustrait à leur activité, on ne pouvait accepter leur enrichissement au prétexte d'accomplir son devoir civique ! Rémunérer chacun à hauteur de ses revenus fragiliserait une collectivité à l'équilibre économique incertain, un gage minimal en éloignerait certainement les plus fortunés (qui ont aussi leur opinion à faire valoir) (applaudissements sur les bancs de droite), sans compter qu'on risquerait 
la corruption - se méfie-t-on jamais assez des puissances de l'argent? Mais les corporations ne valent pas moins que les individus qui doivent pouvoir faire entendre leurs intérêts sans qu'il y soit vu à mal! Bientôt l'amateurisme sembla un danger aussi grand que la professionnalisation.

Les forces du compromis étant à l'œuvre, au bout du jour on finit par s'accorder sur d'excellents principes : quelques (peu mais suffisamment) représentants intègres et frugaux en nombre impair, savants sans pédanterie, compréhensifs quoique résolus, tout à la fois disponibles et indépendants, indifférents à tout intérêt y compris le leur, dont le mandat serait court (sans excès toutefois) mais renouvelable un nombre limité de fois (qui restait à définir). C'était tout et c'était merveilleux. D'ailleurs, cet accord atteint, l'ambiance s'apaisa. On se regarda avec satisfaction, on crut même entendre des soupirs étouffés de soulagement. Hommes et femmes s'embrassèrent, et on n'oublia pas les vieillards assoupis sur les bancs du fond - qui ne comprirent pas, du reste, la raison d'un réveil inopportun au nom de la résolution d'une querelle dont ils avaient oublié la cause. On tenait là enfin la solution constitutionnelle du bien; il ne s'agissait que de la fixer par un vote prudemment remis au lendemain matin.

De retour chez soi cependant, on se mit à réfléchir et la satisfaction fit peu à peu place à la perplexité. Si chacun se reconnaissait dans ce portrait, tous finirent secrètement par espérer ne pas en être. Déjà cinq jours entiers que l'on discutait et les récoltes patientaient, les boutiques étaient fermées, les journaux étaient en sommeil; il n'était jusqu'aux malades dont le sort était maintenu en suspens. On avait cru s'éloigner pour une après-midi, les palabres duraient bientôt depuis une semaine. Encore, pour cette fois, cela demeurait tolérable. Mais à quoi bon se faire élire si c'était pour tout laisser à l'abandon? On ne pouvait décemment s'absenter à nouveau, il y avait trop à faire chez soi - quant à l'intérêt général, d'autres (n'était-il pas présomptueux d'en douter?) s'en occuperaient tout aussi bien. La prospérité générale n'est-elle pas mieux assurée par la réussite particulière que par le dénouement de ces interminables chicanes de boutiquiers? Certes, c'était un devoir moral de déjouer l'anarchie. Mais après tout, l'ordre commençait chez soi. Or, depuis cinq jours seulement les livraisons, les commandes, les bons de retour et ceux à tirer, les rapports à lire et les mémos à rédiger, tout s'accumulait. Dans les campagnes et dans les villes, dans les usines comme dans les écoles on se lamentait : les fruits pourrissaient sur place, les légumes se desséchaient; dans les jardins, la pelouse avait bruni. Et puis on s'indignait 
que les ouvriers ne s'arrêtassent plus de boire, sans compter les enfants qui jouissaient de cette vacance un peu trop bruyamment pour qu'on ne souhaitât pas les en priver. Surtout qu'à force, on les enviait.

Voilà comment s'explique qu'au matin du sixième jour les mines étaient renfrognées. Était-ce trois conseillers pour cent habitants? Un terme de deux ans renouvelable deux fois ou de trois ans renouvelable une fois? On n'écoutait que d'un œil, et ces chiffres lancés à la volée ne furent même pas débattus. Il faut dire que peu comptaient se présenter (encore n'avait-on osé aborder la question des émoluments). Quand il fallut se déclarer, seules quelques mains se levèrent : deux collectivistes repentis et trois légitimistes discrets. Mais cela suffisait, on souffla. Sauf qu'au moment de les ratifier, un troisième ex-collectiviste se déclara, ne supportant pas de voir affaibli le parti de ses anciens camarades. Un ancien légitimiste demanda alors également à en être; il se trouva pareillement un collectiviste pour faire contre-poids... de sorte qu'en peu de temps on comptait une dizaine de volontaires de part et d'autre prêts à se sacrifier à l'intérêt général pour peu que cela lésât le camp opposé. Le vote ne put les départager : il manquait immanquablement à l'unanimité les voix du parti mis en minorité. Bref, tout était à refaire et on était si las... Collectivistes et légitimistes s'en rejetaient à tour de rôle la faute. Peu importait : on ne les écoutait plus, ni ne désirait les voir élus. L'ambiance était morne, il n'y avait plus de vin depuis longtemps. Les sandwichs avaient tourné, l'odeur était infecte.

«Eh bien qu'on les tire au sort!» cria, d'un coup, une voix (c'était une femme qui parlait), et cette proposition sonna comme un coup de semonce sur ces débats qui avaient trop duré. Chacun fut saisi, tétanisé par l'évidence, enrageant en secret de n'en avoir pas eu le premier l'idée. Car voilà qui dénouerait la querelle ! Le sort était impartial, le sort était équitable; il fallait lui remettre le destin de Dédalie. On se décrispa, en apparence, persuadé de tenir en main la solution idéale. Au moment du vote, stupeur : il lui manquait plus du tiers des voix ! C'était à n'y rien comprendre; on se jouait de nous! Et pourtant, c'était ainsi : bien qu'ayant bruyamment protesté de son approbation, personne ne souhaitait être désigné. On avait beau croire à l'arbitraire, on avait peur du hasard.

La discussion s'embourba. Des radicaux voulurent traquer les traîtres au devoir civique : les impudents se découvraient dans l'isoloir, il fallait supprimer le secret du suffrage. On ne les suivit pas. Quelqu'un proposa d'abdiquer l'unanimité pour une majorité des deux tiers, la suggestion 
fit hurler les collectivistes qui avaient dû en ravaler suffisamment, non merci. Par défaut on en voulut à ce diable de père Soureau - n'était-ce pas lui qui, par son idée saugrenue, nous avait mis dans une telle situation? En plus il était assoupi. On le secoua sans trop de ménagement afin qu'il entendît ces remarques perfides. Voici comment il se défendit : il avait le vague souvenir qu'en Allemagne - il y a, oh... bien des années - c'était un poulpe qui décidait des résultats sportifs. On présentait à l'animal deux coffrets de nourriture identiques; s'il optait pour l'un, cela signifiait le succès de l'équipe associée, à moins qu'il ne choisisse l'autre, attribuant la victoire à l'équipe adverse. La nouvelle avait fait grand bruit à l'époque car, aussi curieux qu'il y paraisse, le système s'était avéré infaillible. Et comment ne le serait-il pas? Il alliait sublimité du destin et ingéniosité technique. L'animal était suprêmement intelligent - et probe ! - le procédé ne demandait pas d'entretien. N'était-ce pas d'une simplicité enfantine? Il ne coûtait d'ailleurs rien de l'essayer : le père Soureau, à fins de recherche, s'était justement procuré un spécimen; il proposa qu'on le mît à l'épreuve sur le champ. On le regarda, l'air vide : il paraissait sérieux. On se regarda, perplexe : personne ne trouvait à objecter. De sorte qu'on ne dit rien et que le vieux Soureau alla chercher son animal.

L'ambiance s'était soudain rafraîchie. On feignait la patience curieuse sous couvert d'indifférence attentiste, personne n'osant se dévoiler. À tous, l'idée sembla d'abord fantaisiste et on ne déchiffrait pas bien, sur les visages, si les rictus marquaient la détente, manifestaient un contentement naïf ou se réservaient l'ironie. Mais, à bout, personne n'osait la récuser. Et puis chacun y entrevoyait déjà son intérêt : on n'avait pas d'alternative et on ne pouvait pas continuer sans fin à discuter. Les collectivistes $\mathrm{y}$ voyaient un préservatif contre les dérives fascisantes; les légitimistes le moyen de trancher qu'ils exigeait. Les autres étaient circonspects. Si la dictature de la majorité inquiétait, à tous on n'arrivait à rien. Après tout, si jamais le coup de la pieuvre fonctionnait, on pourrait retourner chez soi. Au pire, c'était arbitraire (mais sans que personne n'ait à en endosser la responsabilité), mais on tenait peut-être en sus un exécutif opératoire qui, quoique représentatif, fût par essence incorruptible. Les Allemands, d'ailleurs, l'avaient éprouvé - c'était assez dire.

Arrivèrent le vieux Soureau et sa fille, transportant un gigantesque bac de verre où remuait un fond d'eau. Le poulpe se tenait tant bien que mal en son milieu. On regarda la bête curieusement, longuement, avec fascination. Elle semblait sereine, déchiffrèrent les amateurs. On n'osait 


\section{Labyrinthe, $n^{\circ} 41$}

pas trop parler. «Eh bien... voilà !» dit Soureau, qui avait préparé les coffrets. Il fallait la mettre à l'essai : quelqu'un proposa de lui demander s'il fallait récuser le principe de l'unanimité. On se récria : puisqu'il s'agissait d'une règle constitutionnelle, le poulpe ne saurait avoir le pouvoir de la défaire. Pourquoi pas la durée de son terme ? L'idée était tentante, mais il fallait une formulation qui fût fermée. Un homme lança, frisant de l'œil : «Qu'on lui demande s'il accepte son office!». On rit d'abord... puis on frissonna. C'était machiavélique et sublime à la fois. Qu'allait-il faire? Et s'il refusait? Où allait et dans quel état errait-on? L'hypothèse glaçait le sang. On n'avait pas d'alternative, mais il fallait admettre que la question tombait sous le sens : elle devait ébranler le système ou le consacrer. Il fallut donc procéder. On vit le vieux Soureau placer, sans trembler, les coffrets de part et d'autre de l'imposante structure, à équidistance du mollusque. En apparence, rien ne les distinguait - ni forme, ni taille, ni couleur. Leur contenu était le même : un reste de chair de crabe de la veille. Soureau annonça que celui de gauche valait «Oui» tandis que celui de droite signifiait «Non». Les dés étaient jetés, rien n'allait plus, on attendit. Dans son aquarium le poulpe ne bougeait pas, semblant se concentrer. On s'avançait, on se pressait, on s'étouffait. Tous les regards étaient braqués sur lui, les parois translucides de l'aquarium se redoublant d'un mur d'yeux globuleux. L'atmosphère devint suffocante, même les sceptiques n'osaient murmurer. C'était pesant. Soudain, on le vit prendre une inspiration; il se décidait. Il cligna de l'œil, imperceptiblement, on entendit un cri : une grosse femme s'était évanouie (d'autres dirent qu'on lui avait marché sur le pied). D'un coup, le poulpe fondit sur sa gauche et de ses habiles tentacules dépeça le coffret. C'était «Oui»; bel et bien «Oui». Quel soulagement!

Et instantanément on se mit à rire, on se congratula, on exulta : le brave animal! Quelle sagesse, quelle résolution! La beauté de son mouvement, la profondeur de sa réflexion; l'irrécusabilité de son verdict; tout inspirait le respect. En un mouvement, il avait imposé sa loi. Par principe ou par habitude cependant, on formula encore une réserve : ç'en était presque trop beau... et si la bête avait été dressée ? Il fallait procéder à un nouvel essai en intervertissant les coffres. Par malice, une femme voulait proposer au poulpe de se récuser. On objecta : c'était absurde, on ne devait pouvoir lui soumettre deux fois la même question. La règle sembla bonne à tous : le danger de contradiction était trop grand, il eût plongé la Dédalie entière dans la confusion (et puis, l'atmosphère étant finalement apaisée, on ne 
voulait plus s'affronter). Comme c'était une règle constitutionnelle, il fallait la voter à l'unanimité - ce fut rapidement fait, à la stupeur générale (on avait perdu l'habitude). Chacun reprit confiance : la démocratie était en marche. Désireux d'éprouver le mollusque, certains proposèrent que le lendemain fût jour de congé; on parla coût du travail; d'autres voulaient envahir la Belgique, ou l'Australie. Un garçon, malicieux, qui avait pris la main de sa voisine et prétendait l'embrasser, demandait l'onction poulpesque. Il fallait un peu d'ordre, et d'ailleurs on risquait de gaver l'animal qui venait juste de se sustenter. Les questions soutenues par le plus grand nombre auraient priorité (l'Australie pouvait bien attendre, la Belgique était trop loin; la voisine en question ne semblait pas vouloir se dérober). Comme tout à l'heure, le père Soureau posa au fond de l'aquarium les deux coffrets parfaitement identiques contenant l'appât. Si la pieuvre optait pour celui de droite, le lendemain serait chômé; s'il partait à sa gauche on travaillerait.

À nouveau, le silence se fit. Et les visages qui s'étaient déridés se fermèrent, stupéfiés par l'enjeu. La démonstration qui suivit acheva de convaincre les sceptiques : un bref moment, le poulpe reposa sur le fond sans plus bouger qu'un mort. Il se fondait au sable, méditatif. Soudain ses tentacules roulèrent de petits cercles, puis de plus en plus grands. Alors que les eaux tourbillonnaient il ouvrit grand ses yeux et, ayant à peine esquissé un mouvement sur sa gauche, il fit brusquement volte-face pour se ruer sur sa droite, se jeter sur le coffret et en dévorer le contenu. «Hourra!», «Vive le poulpe !», « Sus à la représentativité !». La messe était dite : demain, serait congé ! On exulta - cette fois, on en était sûr, la technique fonctionnait. Et à nouveau les chapeaux volèrent, les murs résonnèrent des cris de joie, c'était toute la salle qui tremblait. Quelle ferveur, quel changement! Il y a trois jours à peine, qui l'eût cru? La délivrance pourtant, était complète; on pleurait, on riait, on s'exclamait de concert. Les ennemis d'hier se tenaient par-dessous le bras. On croyait en ce poulpe qui promettait de nous sauver. Pour couronner le tout, certains jurèrent avoir entendu le tonnerre gronder - preuve irréfutable que le ciel même le désignait.

Ainsi, après un vote solennel dont l'issue ne faisait plus de doute, la deuxième loi constitutionnelle désignait Paul, le poulpe, responsable de la législation de Dédalie. La troisième fixait les modalités de son mandat : on ne devait pas l'amener à se prononcer sur une question qui avait déjà était tranchée; les questions lui étaient soumises par ordre de 


\section{Labyrinthe, $n^{\circ} 41$}

priorité; il en traiterait autant que lui permettait son appétit; un assesseur vérifierait la conformité du vote à la constitution (tâche pour laquelle le sage Soureau, homme admirable on n'en n'avait jamais douté, s'était gracieusement proposé). On consigna que Paul avait accepté sa charge dans sa première résolution et décrété férié le lendemain dans sa deuxième. Dans sa troisième, il établissait un salaire minimum, dans la quatrième il baissait les charges patronales. Quant à l'Australie et la Belgique, elle demeuraient dans l'expectative, Paul, probablement repu, refusant d'avaliser la moindre motion. D'ailleurs il était tard et peu importait; après cette journée historique, on leva l'assemblée : Dédalie tenait sa constitution.

Le lendemain, la matinée fut si belle! Le soleil d'été rayonnait, les rares nuages étaient bas, le vent était tombé. On resta chez soi, chacun savourant la paix retrouvée. On se reposait des tensions des derniers jours; on en avait vu tant... La plupart déjeunèrent dehors, on avait ôté ses souliers, les hommes s'étaient laissé pousser la barbe, les femmes fumaient, et vice-versa. Les bêtes paissaient, la mer riait, les oiseaux virevoltaient, la radio jouait sans fin des airs entêtants qui s'élevaient en tourbillonnant vers l'infini du ciel indigo. Là, tout n'était pêle-mêle qu'ordre et volupté; beauté, luxe et calme. On commença à trier ses papiers, on s'affaira au jardin. On savourait la journée en remerciant le bon Paul, à qui l'on savait gré de cette douceur. Une impression nouvelle de sérénité dominait - sans trop savoir pourquoi, on se sentait comme protégé. Rien ne pouvait plus arriver. Les couples se promenant se saluaient et, quoi qu'il fût de saison, on s'émerveillait du beau temps comme d'une chose surnaturelle. On rendait visite aux voisins, emportant des gâteaux dans l'idée de commenter les événements de la veille. La tension, l'atmosphère, la calme résolution du poulpe et la délivrance... on se racontait la scène, que chacun pourtant avait vue! On louait sa sagesse, sa prudence, sa magnanimité. Comme tout le monde les reconnaissait, on ajoutait sans cesse des détails qui donnaient de l'expression. À la fin on les exagéra, c'était à qui avait été le plus impressionné. En un jour, les prodiges de l'animal avaient été démultipliés - les affaires allaient reprendre, les malades se sentaient nettement mieux, déjà les pelouses avaient reverdi. Cette bête avait quelque chose de magique.

Le lendemain et les jours suivants, on commença de lui soumettre ses motions. Il pouvait en traiter de cinq à sept quotidiennement. Dans la quinzaine il limita la journée de travail à huit heures, instaura un jour chômé par semaine, rendit l'instruction obligatoire, réprima le travail 
forcé et l'odieuse pratique du doublage, affirma l'égalité de tous les citoyens. En un mois il avait aboli la corruption, gelé le montant des loyers, rendu partout les bidets obligatoires. Ses résolutions forçaient l'admiration, suscitaient l'éloge : en lui Dédalie se mirait; en ce miroir, elle resplendissait. Il ne fallut que quelques semaines pour que ses avis fussent attendus comme des oracles. Il fournissait leurs sujets aux journaux, qui s'arrachaient. Son nom devenait synonyme de prospérité. Un jour, à la télévision, au présentateur qui lui demandait si Dédalie allait renouer avec la croissance au quatrième trimestre, un fameux économiste en peine de réponse définitive avait lâché : «Paul seul le sait...». La remarque fit naître des sourires de bienveillance, elle appelait sur l'expert l'indulgence - après tout, Paul seul était infaillible. L'expression fit florès : on l'adoptait par complicité, on l'invoquait par humilité, on se sentait d'intelligence. On commença d'appeler l'animal le souverain de Dédalie, surnom qu'il se refusa à officialiser; qu'importe, il resta et on continua à chanter ses louanges. Les poètes l'eurent célébré, les enfants lui envoyaient leurs dessins et des bonbons (il avait décrété le vendredi jour de frites à la cantine). Il pouvait dénouer les questions les plus épineuses et, ses jugements étant justes et mesurés, il parvenait à satisfaire jusqu'aux plus fervents défenseurs des partis les plus opposés. Dans toute la Dédalie, du jour au lendemain on ne mangea plus de poulpe et, par contagion, ni non plus de calamar, de seiche ou de caoutchouc. Ces espèces proliféraient. On voulait que le huit, du nombre de ses tentacules, portât bonheur; de même que le deux de ses mandibules, le un de son auguste tête, le quatre de ses paupières, le six et le dix (selon le nombre de ses tentacules auxquelles on retranchait ou additionnait les coffrets). Le trois et le cinq (qui ensemble formaient le huit magique) étaient d'excellents présages, le sept et le neuf étaient presque parfaits. Le tourteau passait pour un mets de roi. Aux yeux de tous son autorité était indiscutable et c'est tout naturellement qu'on frappa la monnaie à son effigie : de législateur il avait été fait totem, de totem il était devenu le symbole de Dédalie.

Hélas, au bout de trois années de règne Paul mourut - de vieillesse et peut-être d'embonpoint. Quelle ne fut pas l'affliction générale dans toute la Dédalie! On versait de grosses larmes, maudissait le sort, on se lamentait. Quel ne fut le désarroi... Il avait tant de cœurs, il s'était tant donné pour la société, il se souciait tellement de son peuple. On craignit également la vacance du pouvoir (on se sentait si seul tout à coup) : ce 
n'était pas seulement un souverain qui disparaissait, c'était tout le système qu'il portait à bout de bras qui menaçait octuplement de péricliter. On consulta le vieux Soureau qui semblait aussi affligé que las. Il n'avait jamais été commode, avec les années il avait tourné un peu aigri (même si, dans ses tâches d'assesseur, il s'était toujours montré irréprochable). Ce jour-là, comme il paraissait éteint, ce fut sa fille qui parla pour lui. Devenue plongeuse-soigneuse animalière, elle se fit fort de se procurer un nouveau mollusque; on l'en pria, bien qu'elle demandât pour cela du temps qu'on n'avait pas. Pressée de toutes parts elle ramena, dès le lendemain soir, une pieuvre grise de petite taille qu'elle présenta comme le successeur du défunt. Son souvenir était encore vivace, on accueillit celui-là avec un rien de frilosité.

Le deuil national avait été décrété pour trois jours, on avait réquisitionné la grande salle - tout ce qu'il fallait pour examiner à loisir le curieux prétendant. Le poulpe ne fit pas grande impression. D'abord il était terne, alors que Paul chatoyait. Il semblait peu énergique. Ses mouvements étaient rares, et lents. À vrai dire il se montrait carrément amorphe - ce qui, en une occasion si solennelle, jurait. Qui plus est, il n'avait pas encore reçu de nom, ce qui était un comble pour le désigner. À la lueur des plafonniers, ses yeux semblaient un peu hagards; on pressentit qu'il ne serait pas à la hauteur - du moins on le craignait. Le père Soureau amena ses coffrets, un peu renfrogné, et cela valait symbole : c'était toute la cérémonie qui avait perdu de sa majesté. Par rituel ou par défaut, on se résolut à demander au céphalopode s'il acceptait sa charge. On espérait secrètement qu'il arriverait quelque chose d'extraordinaire; qu'il se transfigurerait! Mais la bête resta longuement à reposer au fond de son bac, semblant ignorer ce que l'on attendait d'elle. Comme pour le lui signifier, on souffla fort, s'impatientant. Que voulait dire une telle irrésolution? La fille Soureau expliqua que le poulpe était jeune, et qu'il lui faudrait du temps pour s'acclimater à la captivité. Ce mot rassura. À défaut de l'excuser, il permettait d'expliquer un silence qui semblait se prolonger. Et justement, une heure plus tard à peine, le poulpe se mût, lentement, vers l'un des coffrets. Semi-victoire pourtant : il y avait certes localisé sa pitance, mais peinait à se la procurer. Il s'agita, tourna autour du coffre, le secoua, l'ébranla... et s'en retourna. Puis revint. Puis repartit. Y revenant une nouvelle fois, il en fit le tour (très exactement comme si la clef lui manquait); enfin, se lassant, il s'assit dessus. Cette attitude suscita le commentaire. Certains ne se privèrent pas de le dénigrer. On trouvait un tel suspens déplacé... en somme il exagérait. Au mieux on 
le déclarait peu concerné, mais certains le jugèrent même je-m'en-foutiste. On alla jusqu'à lâcher le mot : «fainéant». Enfin, il glissa en arrière et ses ventouses ouvrirent le coffre auquel elles étaient resté accrochées. Était-il tombé? On se regarda, incrédule, puis l'aquarium : il s'attaquait, comique, au crabe comme si de rien n'était. Alors on murmura : oui...? Un coup d'œil aux Soureau : cela voulait bien dire «Oui»! L'assemblée était saisie - certains, s'esclaffèrent; d'autres se mirent à applaudir, on le félicitait (quand bien même, parfois, certaines dents restaient serrées). Tous semblaient un peu hébétés, mais c'était acté : on intronisa le nouveau Paul (dit «Paul II » ou «Paul le Pataud»), dont le règne s'ouvrait sous le signe de la gaieté, sinon de l'ironie.

Paul II n'eut pas la vie très longue, du coup son souvenir s'est un peu perdu. On sait que son manque d'appétit ralentit le système (au mieux il ne daignait statuer que trois fois par jour), très vite on le critiqua. Ainsi ses offices publics furent peu suivis, surtout parce qu'ils étaient interminables, Paul le Pataud étant d'une lenteur désespérante. Enfin, il n'était pas très photogénique et les journaux évitaient son portrait. On s'était à peine habitué à lui qu'il succomba : il n'avait pas même fait deux mois ! Mais son décès fut atroce et laissa un goût amer. Ses dernières décisions avait été mauvaises - il avait coup sur coup augmenté le tarif des autoroutes et de la collection des déchets, s'était mis à dos les taxis, avait relevé le taux directeur de la banque centrale et mécontenté les coiffeurs, il avait même refusé l'établissement d'un musée «Paul Ier» (qui, pour sa part, eût pris soin d'offrir des contreparties). Au lendemain on le retrouva mort, gisant dans un flot d'encre, l'estomac, perforé par un ulcère, en état de décomposition avancé. On fut pris de remords, la défiance publique l'ayant probablement meurtri. Et puis, l'héritage de son auguste prédécesseur devait avoir été trop lourd à porter; bref, on l'avait sans doute hâtivement jugé. Et puis surtout, le père Soureau démissionna, ce qui fut un choc. On ne le savait pas si sentimental, le geste surprit autant que sa soudaineté. Sans compter que l'on ne s'était pas préparé à se passer de lui. Malgré le peu de conviction de sa dernière trouvaille, sa fille, à nouveau, fut commissionnée. Elle présenta des excuses circonstanciées pour Paul II, certes un peu apathique mais à qui l'on avait sans doute trop tôt trop demandé. On acquiesça, penaud. Elle demanda à nouveau du temps pour ménager la transition, sauf que cette fois elle fut exaucée et on ne fut pas déçu : il ne lui fallu que quatre jours pour présenter Manon, un mètre cinquante pour soixante-dix-huit kilos, dauphin. 


\section{Labyrinthe, $n^{\circ} 41$}

Avant tout, le public fut estomaqué. On s'attendait légitimement à un nouveau poulpe, qu'est-ce donc que cette fantaisie signifiait? On parla d'atteinte à la constitution; pire : c'était un outrage à la mémoire du bon Paul! Comment osait-elle proposer un cétacé pour présider au destin de Dédalie ?! La foule était furieuse que la fille Soureau avait fait rassembler au parc aquatique. C'était plus qu'outrepasser son mandat : c'était l'usurper. Ses mots d'introduction furent inaudibles, couverts par les réactions indignées. Elle prétendit tout de même à une démonstration des talents du mammifère, à qui, plantée au milieu du bassin, elle présentait deux cerceaux tenus à bout de bras. Au signal, l'animal se mit à foncer, jaillit brusquement hors des eaux et, en un instant, franchit le cercle de droite. À peine retombé, il continua sa nage à toute allure, se retournant, frétillant, couinant; il en était tout agité. Au nouveau signal l'animal répéta sa performance, optant cette fois pour le second cercle, bravo, après quoi il stationna un moment - nageant à la verticale, à demi immergé, avant de se laisser retomber en arrière. Il claqua à nouveau de son museau.

Les bouches étaient bées. La foule, perplexe, semblait certes apprécier le spectacle mais elle ne savait pas comment réagir : on n'en comprenait pas l'intérêt politique. La fille Soureau traduisit : c'est que les cerceaux pouvaient figurer les coffrets ! À cette réalisation, les citoyens demeurèrent un instant incrédules avant que les mains, d'instinct, ne se missent à battre... et en un éclair, ce fut une ovation terrible : on acclamait Manon, nouvelle responsable de la législation de Dédalie.

Pendant un demi-siècle, les habitants de Dédalie admirèrent à l'aquarium leur souveraine delphinidé. Pour cent kilos de sardines par semaine, elle tranchait les litiges et les indécisions, décrétait, ordonnait, jugeait, savait prononcer une résolution, prendre des arrêtés, rouler sur sa queue, et resta appréciée des Dédaliens, - qui cependant n'étaient pas des citoyens conciliants. Elle se montrait une législatrice infatigable : du matin jusqu'au soir, à raison de trois cent quarante-cinq jours ouvrables par an, elle tenait séance publique et statuait sur toutes sortes de questions, se prononçant de deux cent cinquante à trois cent vingt fois par jour selon les périodes et les nécessités.

Une telle ferveur législative amena certes son lot d'ajustements. On avait décuplé le nombre de ses assesseurs, le relevé de ses innombrables décisions (pas loin de cinq millions) occupait des volumes entiers. Assister aux consultations de Manon devint une occupation très prisée - on conviait parents et voisins, amis et enfants aux délibérations du jour, voire pour 
l'après-midi seulement (les sessions à l'heure du pique-nique ainsi que la séance dans la matinée du mercredi, particulièrement, étaient très appréciées) - et c'était merveille de voir chacun commenter ses performances, l'encourager, se prendre de passion pour la vie de la cité. De sorte qu'on crut manquer de points à délibérer et le spectre de cette pénurie de questionnements allait bientôt menacer jusqu'à l'équilibre du système juridique : on craignit et de faire retomber l'enthousiasme citoyen qui s'était emparé du peuple de Dédalie, et que les effets s'en ressentissent sur une Manon dont l'ardeur civique provoquait l'admiration - n'était-ce pas l'indifférence publique qui avait désespéré Paul II jusqu'à l'abattement? Heureusement, il n'en fut rien.

À mesure qu'ils constataient l'efficacité délibérative de leur souveraine, les habitants de Dédalie redoublaient de points à lui soumettre. On l'amenait à déterminer toutes sortes de choses - les normes à établir et les us à abroger, la litanie de régulations à préciser, la forme des bâtiments et la composition des parfums, les mesures pour les vestons et la taille des haies, la longueur des cérémonies et la couleur des fairepart, toute question valait promesse de saut virevoltant, présageait une cabriole, dans tout choix se devinait une figure d'acrobate. Les débats publics se privatisèrent : on venait voir Manon trancher pour soi, ce qu'on mangerait le soir et que faire des enfants, l'heure à laquelle on déjeunerait dimanche et s'il fallait inviter mémé; tout prenait force de loi. «-Te verrais-je ce soir?» demandait l'amant transi, «-Si Manon le veut...» répondait naturellement sa dulcinée. Et ils se précipitaient tous deux à l'aquarium, le cœur battant. Elle était d'une intelligence vive, se montrait toujours proche de son peuple et exerçait avec discernement son pouvoir sur tous les chapitres de leur vie. De sorte que Manon en vint à totalement épargner à ses sujets les affres de la décision.

En peu de temps, on se montra incapable de se prononcer sans la consulter - la fiabilité d'un équipement, d'un matériau, d'une construction; la concordance d'un habit, le motif des rideaux, le style des franges et la vocation du petit dernier (avocat ou pompier?). Confronté à deux hypothèses formellement comparables, qu'est-ce qui permettait de trancher? Opiner fatigue, exige compétence, patience et réflexion (sans compter, peut-être, un peu de chance) tandis que déléguer ne relève que du bon sens : Manon est perspicace, sa bonté n'est plus à démontrer et surtout elle n'est pas sujette au doute. Et puis, le cas échéant, on pourra toujours se laver les mains sur elle de ses infélicités. Bref, au moindre dilemme, 


\section{Labyrinthe, $n^{\circ} 41$}

elle était le compas moral auquel recourir; si l'on devait vraiment savoir, c'est à elle qu'il fallait demander.

Non que ses décisions fussent toutes irréprochables. Manon, dauphine espiègle qui mettait toute sa fougue à la tâche, ne manquait pas d'y introduire parfois un peu de sa malice : les plombiers se demandèrent longtemps comment serrer leurs boulons dodécagonaux (résolution $\mathrm{n}^{\circ}$ 400.374) avec une clef devant légalement compter huit côtés (résolution $\mathrm{n}^{\circ}$ 77.809); on ne comprit pas bien comment appliquer la réglementation sur la consommation des lottes (résolution $\mathrm{n}^{\circ} 222.456$, elles doivent être cuisinées entières mais, résolution $\mathrm{n}^{\circ} 450.112$, avoir été vendues sans leur tête); pourquoi, sur les automobiles, les compteurs peuvent afficher jusqu'à $260 \mathrm{~km} / \mathrm{h}$ (résolution $\mathrm{n}^{\circ}$ 99.654) quand la vitesse maximale est de deux fois moins élevée (résolution $n^{\circ} 1.895 .957$ ) et aussi comment les odeurs de transpiration pouvaient se voir bannies du réseau métropolitain (résolution $\mathrm{n}^{\circ}$ 665.948) puisque les crédits pour la construction d'escaliers mécaniques n’avaient pas été débloqués (résolution n 665.949). Les incidents se multiplièrent qu'on était bien incapable de trancher. Il fallait battre en retraite - ralentir, renoncer au poisson et laisser fuir les robinets : l'esprit supérieur de Manon avait ses raisons que l'on ignorait. On se rendait dans le métro comme on aurait voyagé dans une province putride. On se rêvait en Europe. On se croyait à Paris.

À force, la perplexité gagna. «- Combien font 2 et 2 ?» demandait la maîtresse; «- Manon seule le sait...» se décourageaient les élèves, à qui ces subtilités mathématiques échappaient. Ils avaient raison, les certitudes manquaient. On se permit des entorses, croyant éviter les conflits. Mais ils renaissaient ailleurs et chaque nouvelle décision apportait son lot d'aménagements contrariants. La suspicion gagna. On se défiait des voisins qui se rendaient quand même un peu trop souvent au parc aquatique. Les familles s'installèrent de plus en plus loin les unes des autres, afin d'éviter les embêtements. On ne se communiquait plus que des informations insipides, évitant soigneusement tout avis trop prononcé - il n'était plus question que de l'appétit des bêtes, du visage de la mer, du tournoiement des oiseaux dont, pas plus que des programmes radiophoniques insipides, on n'osait se plaindre. On parlait du temps et, quand il était beau, on s'en émerveillait comme d'une chose surnaturelle. La bonhommie avait disparu, les avocats proliféraient.

Au fil des années, on fit à la fille Soureau bien des réflexions à ce sujet qui finirent par la vexer. Elle se retira loin des Dédaliens et les 
méprisant un peu, pestant sur leur manque de reconnaissance et raillant à demi-mot leur imbécilité. Elle-même vieillissante, elle repensait à son père, disparu depuis bien des années et dont on s'accordait à dire qu'il fut le meilleur assesseur de l'histoire de Dédalie. Ce n'est pas sans réjouissement qu'elle lut dans son journal qu'il prenait bien soin de s'assurer contre le sort en versant quelques gouttes d'arsenic dans la nourriture offerte au sein du mauvais coffret. Cette découverte la fit sourire, elle lui fournit également le motif d'un tour à réaliser. Ainsi, dans sa résolution $\mathrm{n}^{\circ}$ 4.743.754 Manon désignait par avance, conformément au vœu de la fille Soureau, une méduse pour lui succéder. Au moment venu il fut donc procédé ainsi et, puisque c'était la loi, on désigna Alexandre, rhopilema esculentum de la plus belle espèce, pour présider au destin de Dédalie à la suite de la dauphine.

Cela n'alla pas sans peine, et il fallut bien du temps pour s'y faire - Alexandre étant infiniment moins brillant (on le dit pudiquement «Placide») que l'espiègle Manon. Il paru surtout plus mesuré - sa nage étant moins prompte, plus réfléchie, énigmatique. Ceux que le sujet intéressait encore louèrent d'abord sa circonspection, avant d'en être un peu frustrés : la sagacité de ses interprètes se reporta sur les circonvolutions de son mouvement, le frémissement de ses tentacules, les intermittences intrigantes de ses pâleurs matutinales; le moindre battement de son ombrelle provoquait toutes sortes de conjectures. Mais les penchants oraculaires de l'hypnotique animal finirent par les lasser eux aussi. Plus rien ne se décidait et on n'invoquait plus le nom d'Alexandre que comme un présage, une promesse, une menace abstraite; accolé à une décision hypothétique située dans un futur lointain. L'effigie de la méduse qui avait été gravée au fronton des édifices enveloppait la chose publique d'une atmosphère de mystère, laissant flotter dans toute la Dédalie comme un parfum de mythe. Sa souveraine apathie transpira bientôt dans tout le pays qui vivait dans l'attente de ses lumières prophétiques. À moins qu'il ne les ignorât tout à fait.

Je ne sais pas exactement ce qu'il en est aujourd'hui, j'ai entendu dire qu'après bien des flottements on s'en était accommodé. Les Dédaliens ont largement prouvé être un peuple de ressources, j'imagine qu'à force ils s'y sont rangés et que leurs usages ont évolué. J'avoue surtout ne pas savoir comment a été adapté le procédé technique des coffrets. D'ailleurs je ne sais à peu près rien des méduses; si ce n'est leur exceptionnelle longévité. Et, comme tout le monde, qu'il s'agit du seul animal dépourvu de cerveau. 



\section{Salut François,}

Comment vas-tu? Comment va la France? Tu la supportes encore? Je ne sais pas comment tu fais, franchement... Moi je n'en pouvais plus, et ce n'est pas seulement à cause de ce que m'a fait subir Virginie. Tout me pesait, le baratin des ministres comme la conversation de ma bellemère. J'avais du mal à saisir exactement pourquoi. Vu d'ici je commence à mieux comprendre : en France, tous les problèmes politiques, sociaux, existentiels, économiques, etc., sont posés en termes de morale. Est-ce que c'est bien? ou est-ce que c'est mal? Du coup ils ne sont pas posés du tout. Ni réglés, par définition. Mais passons.

De mon côté, bonne nouvelle : j'ai trouvé du travail. C'est un peu spécial, mais c'est très correctement payé grâce à un système de primes indexées sur mes résultats. Je bosse pour une grande clinique spécialisée dans l'euthanasie. Heureusement, mon job ne consiste pas à faire des piqûres, ni à administrer le moindre traitement (tu me connais, tu imagines bien comme je détesterais ça). Non, c'est un pur boulot de commercial. Je suis chargé de recevoir les gens, pour leur présenter toute notre gamme de services. Et je peux te dire qu'elle est étendue : euthanasie active directe, active indirecte, passive, suicide assisté, notre clinique propose de tout. Ca n'est pas un hasard si on est leader sur le marché.

Je ne vais pas te détailler l'ensemble de nos produits, mais l'idée générale est de faire en sorte qu'il y en ait pour tous les goûts. Il y a évidemment la mort ultra-rapide, qu'on administre soit avec une piqûre, soit avec une boisson sucrée ou alcoolisée, soit avec un bonbon qu'on avale (les gens choisissent plutôt la boisson, je ne sais pas pourquoi). C'est la formule qu'on appelle «Socrate style». Mais il en existe d'autres pour les amateurs de sensations fortes : le «Kurt Cobain style», par exemple, ou même le «James Dean style» (on a des accords partenaires avec un constructeur automobile qui nous prête sa piste d'essais, nos clients peuvent l'utiliser pour se crasher en voiture). Il y a aussi la possibilité de «faire durer le plaisir», comme disent nos prospectus, en s'adaptant aux désirs de chacun. Comme c'est du sur-mesure, c'est facturé plus cher, et parfois même beaucoup plus cher, mais ça permet de quitter cette vie tout doucement, en faisant quelque chose qu'on aime bien : manger un homard à l'armoricaine (ça arrive), regarder une dernière fois «la Vie de Brian» 
(un type m'a demandé ça l'autre jour), profiter des services sexuels d'un de nos agents (c'est notre offre «Félix Faure», elle marche très bien).

La boîte a un bon slogan : "Chez nous, la fin justifie vraiment les moyens ». Et en effet, la mort peut être conçue comme le moment de vivre enfin son fantasme le plus inavouable : ça va de participer à une orgie jusqu'à sauter en parachute sans parachute. Si quelqu'un rêve de savoir ce que ça fait de tuer quelqu'un, on lui offre même la possibilité de le faire (il suffit de trouver un client qui est d'accord pour mourir comme ça), avant d'être tué à son tour par un autre, etc. Mais je crois que ce qui plaît le plus, c'est qu'on a bâti notre image sur l'écoute du client : il faut bien se rendre compte que celui qui vient nous voir ne sait pas toujours exactement ce qu'il veut; on prend donc le temps qu'il faut pour lui proposer ce qui lui correspond, en s'engageant à un maximum de créativité et d'efficacité. Rien à voir avec les traitements froids et impersonnels dispensés dans les cliniques aseptisées qui occupent le reste du marché.

Ça donne parfois de drôles de résultats, mais c'est bien, c'est varié, on ne s'ennuie pas. Tiens, hier, une femme est arrivée avec un livre de Julian Barnes sous la main, celui où il dit avoir été tenté de mettre fin à ses jours avec «un bain chaud, un verre de vin à côté des robinets, et un couteau à découper japonais à la lame particulièrement aiguisé». Elle souhaitait la même chose! Elle l'aura dès demain.

Je t'embrasse fort

Paul 


\section{Grandeur et décadence du Pannoon}

Aux grands cerveaux, Dédalie reconnaissante. L'inscription avait été gravée sur le fronton du bâtiment aux dimensions monumentales. La visite des lieux n'était plus libre, depuis qu'une commission d'enquête avait conclu à un lamentable écart entre les premières espérances du projet et ce qu'il donnait à voir. Ah ! que l'idée initiale était belle! Il s'agissait de mettre en œuvre les magistrales avancées de la neuroscience et de l'intelligence artificielle, de ressusciter la pensée des plus grands cerveaux. Des écoliers en troupe viendraient au Pannoon et s'entretiendraient avec les plus brillants esprits de la veille, maintenus pour toujours en activité sur un support sec (un ordinateur) ou un support monillé (une espèce de cervelle de synthèse). Avant l'inauguration du lieu, un code de l'honneur avait été prescrit (il était ainsi interdit aux écoliers de soumettre aux cerveaux mathématiciens le problème qu'ils avaient à résoudre en classe) et une première liste de candidats à l'immortalité cérébrale avait été dressée. L'édifice avait coûté une fortune, et les meilleurs cyber-artistes avaient décoré la voûte d'images électroniques changeantes, représentant tel connectome et tel circuit intégré. La fondation qui était à l'origine des techniques de transfert entre les routes synaptiques avant décès et leurs contreparties post mortem avait assuré que tout était au point. Le cas test du chien Fido, - dont le cerveau, embaumé puis transféré après sa mort (naturelle) sur un disque dur, avait émis une série d'aboiements reconnaissable du premier coup par son maître (d'ailleurs président d'honneur de ladite fondation) - avait balayé les dernières hésitations.

Hélas, des difficultés en rafale ralentirent la marche du progrès. Tout d'abord, l'espérance de vie étant impressionnante en Dédalie, les grands cerveaux n'étaient pas pressés de s'éteindre, condition néanmoins nécessaire et préalable à leur résurrection. Ensuite, une malencontreuse panne de secteur avait gâché les efforts de préservation de la première biologiste géniale qui s'était décidé à mourir. Lorsqu'on eut enfin obtenu plusieurs beaux cerveaux ranimés et que fut inauguré le Pannoon, une gêne s'installa. Les merveilleux esprits d'hier n'avaient guère de conversation. On leur posait une question, et venait une réponse assez stéréotypée, qui paraissait sortir d'un choix entre quelques phrases possibles. Pour le poète, cela passait à la rigueur. On obtenait de lui des variations de ce type : la salamandre n'a aucun mérite ; la vision du dernier dodo périssant; 
c'est la fausse et sainte face de l'un des fils du feu. Les spécialistes de l'œuvre déclarèrent que ces sentences étaient typiques du style elliptique et du ressassement opératoire de l'écrivain. Admettons. Quand la grande physicienne se cantonnait à des réponses comme encore des navets ou c'est moi qui le méritais ce prix, il s'avérait toutefois plus difficile de justifier la pauvreté ou la circularité de ses énoncés. Le responsable de la fondation expliqua que notre fonctionnement cognitif étant fondé sur la répétition, il devenait raisonnable d'estimer que les plus grands cerveaux fussent encore plus répétitifs que les autres. Les réseaux sociaux apportèrent une autre hypothèse, selon laquelle «les génies au fond ils sont comme nous ». La grande voûte cessa rapidement d'attirer les foules. Les gamins se lassaient d'entendre même si je le savais, je ne vous le dirais pas dans le synthétiseur vocal du cher professeur disparu. Bien des visiteurs s'offusquaient de recevoir les bordées d'injures que lançait le cerveau du compositeur majeur au milieu de quelques mi sol mi sol $d o$. Le récit d'une scène d'enfance avec une toupie transparente perdait de son attrait à la cinquantième écoute.

La commission d'enquête ne fit qu'entériner ce que tout le monde avait plus ou moins deviné. La conception scientifique de la pensée en Dédalie était si pitoyablement incomplète qu'elle ne soutenait qu'une vue tronquée de l'intelligence. Quant aux prodigieux moyens techniques, ils ne suffisaient pas à fixer plus de quelques séries de mots et d'idées, qui, au fond, n’intéressaient personne. Le Pannoon ferma ses portes au grand public et devint un sépulcre voûté, comme une gigantesque boîte crânienne sombre et déserte, abritant des intelligences mi-rescapées et mi-détruites, forcées d'ânonner en boucle les vestiges insignifiants de leur penser disparu. Et la voix artificielle du poète disait par instants : approche ton oreille de mon crâne, tu entendras la mer. 


\section{Hello François,}

Désolé de ne pas t'écrire davantage. Le boulot me prend beaucoup, je n'arrête pas. En fait de dimanche, j'ai droit à deux demi-journées de repos chaque semaine : le mardi matin et le vendredi après-midi. Ce n'est pas idéal pour se changer les idées, mais tant pis. Au moins, je n'ai pas le temps de m'ennuyer.

J'ai quand même trouvé l'occasion de recroiser Georges, tu sais, le francophone sympathique dont je t'avais parlé dans ma première lettre. Un drôle de type. En fait, je me rends compte que la vie ici ne lui plaît pas du tout. J'ai beau lui opposer que chacun peut manger ce qu'il veut, baiser comme il veut, mourir comme il veut, lui ne jure que par Bernanos : «la Liberté pour quoi faire?» Il trouve qu'à force de pouvoir faire plein de choses, les gens n'ont plus le temps de réfléchir à rien. Il est scandalisé par une pub pour je ne sais plus quelle marque de fringues, que tout le monde cite à tout propos et qui résume assez bien l'état d'esprit général : «Faites plaisir à vos envies».

Mais il exagère, ce Georges. C'est bien agréable, la vie ici. On garde l'impression que tout reste possible. Je ne t'envie pas d'être resté en France.

Avec toute mon amitié,

Paul 



\section{Mon cher Fanfan,}

Merci pour ton mot. Je suis content d'avoir de tes nouvelles, même s'il est désolant de penser qu'un type aussi intelligent que toi est toujours au chômage. Tiens bon!

Mais tu me dis que la description de mon nouveau boulot te choque un peu. Encore une fois, si ça peut te rassurer, je ne suis pas du tout rattaché à l'équipe exécutive : moi je suis juste un commercial, chargé de vendre à nos clients un service personnalisé. Évidemment, il vaut mieux aimer les gens, le contact, tout ça. Mais tu sais que c'est mon cas. Je tiens d'ailleurs à préciser que je ne leur force jamais la main. Au contraire : si je sens que quelqu'un hésite, je l'encourage à prendre le temps de la réflexion. Ca fait même partie de notre stratégie marketing. Car on a beau faire de la pub un peu partout, dans les maisons de retraite comme sur les sites internet destinés aux jeunes cadres surchargés de travail, on sait très bien que rien ne vaut le bouche-à-oreille pour convaincre les gens de venir chez nous plutôt qu'ailleurs. Il faut qu'ils se sentent en confiance. D'où l'intérêt de laisser certains clients repartir tranquillement chez eux après nous avoir consultés. Ils le racontent à leur entourage, ça améliore notre réputation. Et puis de toute façon, «la mort rattrape ceux qui la fuient », comme disait Horace.

D'autre part, puisque tu sembles avoir des doutes là-dessus : oui, l'activité de la clinique est parfaitement légale. Tout repose sur un principe de base, qui est en vérité assez indiscutable : chacun est libre de mourir quand il veut et comme il veut. Malade ou pas. Au début, quand il a été question de l'inscrire dans la loi, il paraît que des comités d'éthique et des associations religieuses se sont mis en place pour protester. Il y a même eu un grand débat entre économistes et sociologues : les uns craignaient un impact négatif sur la croissance; les autres faisaient valoir qu'au contraire, ça créerait des emplois (comme celui dont je bénéficie) et, surtout, que ça serait très sain pour l'ensemble de la société. Pour bien comprendre le raisonnement, le plus simple est de faire un parallèle avec la vie biologique du corps : le corps ne fonctionne bien que s'il sait éliminer les cellules peu performantes. Au fond, c'est la même chose : une maladie qui se complique, ou même un chagrin trop fort, ça rend incapable de travailler correctement, de consommer normalement, de vivre à $100 \%$. Là, avec l'assistance organisée au suicide, c'est réglé. 


\section{Labyrinthe $n^{\circ} 41$}

Et finalement, ça a même un autre effet intéressant, d'après un article que j'ai lu hier : figure-toi qu'il n'y a pas tant de gens qui sautent le pas. Comme si savoir qu'il est leur très facile de se supprimer leur suffisait. Par ailleurs, pardon de revenir à des considérations bassement économiques, mais ça a relancé toutes sortes d'entreprises concurrentes : des psys, des labos pharmaceutiques spécialisés dans les antidépresseurs... Tout ça, ça crée de l'emploi mon vieux ! Je ne te parle même pas des agences publicitaires qui rivalisent de créativité pour fourguer les nouveautés. Tiens, ces jours-ci, les radios diffusent ce message en boucle par exemple : «Vous vous sentez à plat? Pas une raison pour crever : ayez le réflexe Rustinal».

Allez, pour achever de te convaincre que mon boulot n'est pas si abominable, je te laisse méditer ces quelques lignes. Elles sont imprimées en vert sur toutes nos brochures. Mais attention! elles ne sont pas de n'importe qui. Toi qui aimes la philo, ça devrait te plaire. C'est du Sénèque, Monsieur :

«Si je puis opter entre une mort compliquée de tortures et une mort simple et douce, pourquoi ne prendrais-je pas cette dernière? Tout comme je fais choix du navire, si je veux naviguer; de la maison, s'il me faut un logis, ainsi du genre de mort par où je voudrais sortir d'ici. Et de même que la vie n'en est pas meilleure pour être plus longue, la mort la plus longue est la pire de toutes. La mort est la chose où l'on doit le plus agir à sa fantaisie : l'âme n'a qu'à suivre son premier élan : préfère-t-elle le glaive, le lacet ou quelque breuvage propre à glacer les veines, qu'elle achève son œuvre et brise les derniers liens de sa servitude. On doit compte de sa vie aux autres, de sa mort à soi seul. La meilleure est celle qu'on choisit.»

Au moins, ici, les marketeurs ont des lettres, non?

Bises,

Paul 


\section{Homeless Star Ac'}

\section{Chers tous,}

Pardonnez-moi ce message collectif, je prendrai le temps d'écrire à chacun au fil de mon séjour. J'ai assisté hier à une cérémonie d'un genre un peu particulier. Ils appellent cela «Homeless Star Ac' », vous comprendrez bientôt pourquoi. Comme certains d'entre vous le savent peut-être, le solstice d'hiver est ici aussi l'occasion de réjouissances exceptionnelles. On achète beaucoup et l'on offre presque autant (pas aux mêmes, certes : certains y gagnent sûrement, mais personne ne semble y perdre). Ce qu'il y a de particulier, c'est qu'on donne la possibilité aux plus misérables de s'arracher d'un seul coup à leur condition. Voilà comment on procède : il y a un an que des limiers ont parcouru la Dédalie à la recherche des meilleurs mendiants du pays. Meilleurs? Oui, ceux qui présentent le mieux. À savoir : ceux à qui le dénuement n'a pas enlevé le sens de l'humour, de l'élégance verbale (pour la vêture, on sait être indulgent), de la présentation de soi. Certains les appellent les gracieux, d'autres les graciés. À vous de trancher. En tout cas, ils sont sélectionnés en fonction de la pertinence, de la cohérence et de l'harmonie intrinsèque de leur discours - la condition sine qua non de la sélection étant un minimum de décence physique (les mutilés, couperosés ou atteints d'eczéma ne sont pas acceptés, et c'est dommage, car il y a parmi eux d'excellents orateurs). Il y a six mois, une dizaine d'entre eux s'est ainsi retrouvée au «Château », à savoir un hôtel particulier sis en plein centre-ville, où ils ont pu s'entraîner à mendier jusqu'à la perfection pour atteindre un niveau maximal lors de la nuit du solstice. Cet hôtel n'est pas chauffé, il ne s'agit pas d'habituer les gueux au confort, et ils en sont expulsés tous les soirs pour retrouver dehors la réalité rugueuse qui a fait leur spécificité. Pour diminuer les risques de violences qui risqueraient d'éliminer prématurément certains candidats, ils sont munis d'un badge électroniquement relié aux services du Château qui émet un signal en cas d'agression. Les services d'ordre de Homeless Star Ac' peuvent ainsi intervenir rapidement et mettre hors d'état de nuire le malfaiteur susceptible d'entraver le bon déroulement des festivités. Pour éviter la déperdition complète des forces, une soupe hebdomadaire est servie dans la cour du château, mais la nourriture du restant de la semaine est aux frais des candidats, afin qu'ils ne perdent point le sens des réalités. Pendant la journée, ils sont 
conseillés par des experts : anciens mendiants, acteurs, hommes politiques - difficile parfois de les discerner, d'ailleurs. On améliore leurs effets de manche, leur crédibilité, on aiguise leur sens de l'adaptation au public, la compréhension de ses attentes, le sens de la surprise, du prévisible bien dosé. Les séances d'entraînement sont retransmises régulièrement, si bien que les candidats sont désormais facilement reconnaissables dans l'espace public. Mais il est interdit de les aborder en tant que stars. Il faut les traiter en tant qu'homeless. C'est la règle qui pimente l'ensemble. Tout cela sonne bien sûr un peu faux, tout le monde le sait, mais tout le monde joue le jeu. Depuis une semaine, les candidats ont été libérés et ont retrouvé leur mode de vie antérieur. Leur badge les protège et l'on n'a pas eu à déplorer d'incident cette année. Le froid et l'urgence de la victoire augmentent naturellement l'inventivité, on pouvait s'attendre à ce qu'ils donnent le meilleur d'eux-mêmes.

L'une des difficultés pour les organisateurs est le sort réservé aux mendiants lambda. On a vu en effet des individus perturber le bon fonctionnement de la cérémonie en se livrant à des performances dignes des meilleurs candidats le soir même de l'évènement. Pour éviter cette concurrence déloyale, la milice a cantonné au réseau du métro l'espace de mendicité officielle, reléguant pour une journée les non-candidats à la surface. Ne vous méprenez pas : ce n'est pas une chape de plomb totalitaire qui s'abat sur ce pays, c'est juste pour une journée, pour que la fête se passe bien, pour que les performants ne soient pas gênés, que le public en profite et que les organisateurs en jouissent. Le lendemain, tous les mendiants pourront à nouveau mendier où ils l'entendent et comme ils le désirent. Il fallait les voir, les vrais, appliqués, concentrés, récitant leur partition devant un parterre de voyageurs suspendus à leurs lèvres toujours un peu faméliques. Je ne voulais en rater aucun, j'ai dû bondir de rame en rame. J'ai pu voir ceux que j'avais ratés sur les multiples écrans disséminés dans les couloirs du métro. Je les ai trouvés tous bons. J'ai donné à chacun, en acte ou en pensée, toujours avec sincérité. Le public semblait plus sceptique, chuchotait des comparaisons avec les années précédentes, se plaignait que «le niveau baisse» etc. Mais tout le monde regardait, écoutait ou filmait. À six heures tapantes, il fallut voter, au sortir du métro, dans un bureau de vote préfabriqué. Les candidats, pendant le dépouillement, étaient logés au Château pour y prendre un dernier vin chaud. À huit heures, proclamation des résultats. Il paraît que les vaincus, furieux, ont été expulsés par la milice, je n’ai vu à la 
télévision que le vainqueur ému et interviewé. Il a dit vouloir utiliser la somme impartie à des fins citoyennes, pour conseiller par exemple de futurs compagnons d'(in-)fortune, ou entrer dans la prestigieuse Académie de Prostitution. Les résultats étaient commentés télévisuellement par des experts ou des personnalités, les membres du jury bien sûr mais leurs amis et collègues également. «Je l'ai trouvé excellent, c'est amplement mérité, on sent que ce monsieur vient de loin et qu'il ira loin.» «J'avoue ne pas partager votre enthousiasme, cher A., tant le malheureux candidat de la ligne 6 m'avait semblé supérieur. Langage populaire, appel sans pathos à une commisération raisonnable, évitement de la culpabilisation, gaudrioles salutaires, tout y était. Le vainqueur a utilisé des imparfaits du subjonctif pour quémander, était bien trop flegmatique, bourgeois même. Ca ne rime à rien.» «Vous préférez les candidats qui mendient en verlan ou en rap? Quel exemple voulez-vous qu'ils donnent?» «Je ne préfère rien, je constate.» «Et vous, cher M., vos analyses?» «Les Dédaliens ont compris qu'il fallait un souffle nouveau dans les représentants du Lumpenproletariat, ils l'ont trouvé. C'est un message clair, il va falloir en tenir compte.» Etc.

Et les perdants? La vie sera dure dit-on, ils souffriront à la fois du retour à l'anonymat et de leur célébrité. On les reconnaîtra longtemps après la soirée fatale, mais on tolérera mal leur tentative de numéro, on reconnaîtra leurs trucs, les procédés enseignés par leurs coaches. On leur donnera moins, voire plus du tout. Leurs rares soutiens indéfectibles n'oseront plus les applaudir. De toute façon leur amertume nuira à leur compétence. Il ne serait pas étonnant que le Château soit un jour pris d'assaut, pillé ou brûlé, comme les Tuileries. Au moins, les Tuileries étaient un lieu de fête. De toute façon, le Château est de moins en moins surveillé, l'audience de l'évènement étant en baisse depuis plusieurs années. Les gueux n'y trouveront pas grand-chose. Il vaudrait mieux qu'ils se renseignent sur la résidence des organisateurs, si tant est que je sois bien placé pour leur donner des conseils.

Voilà un aperçu des coutumes de cette contrée, je vous en dirai plus dès qu'un phénomène singulier me semblera digne d'être relaté. Portezvous bien et marchez à l'ombre.

Philarète Aporos 



\section{Mon bien cher François,}

Comment vas-tu? Tu ne me donnes plus de nouvelles. J'espère que c'est le signe que tu as enfin trouvé du travail, au moins. J'espère surtout que tu n'es pas fâché contre moi, et que tu vas pouvoir recommencer à m'écrire. Tu me diras ce que tu deviens. Tu me diras aussi ce qui se passe en France, et comment tu vois les choses évoluer. J'ai hâte. Nos conversations d'autrefois me manquent un peu, tu sais.

Je pourrais me renseigner moi-même, ceci dit : les médias ici ont un fonctionnement assez idéal. Non seulement tous les sites d'informations sont gratuits, mais il y a une liberté d'expression absolument totale. Aucune censure, aucun politiquement correct, aucun dogme. Rien qui ressemble de près ou de loin à notre loi Gayssot, tiens. C'est aux historiens d'écrire l'histoire, point. Comme ça, chacun a le droit de se faire son opinion, et de l'exprimer. De penser ce qu'il veut, si tu préfères. Personnellement, je trouve ça plutôt sain. Et puis les tarés, quand ils sont privés de la posture du martyr, semblent tout à coup beaucoup moins séduisants.

De toute façon, cette liberté d'expression ne pose pas vraiment de problème : grâce aux algorithmes de Google et à la puissance des annonceurs publicitaires, l'information repose sur le principe des contenus personnalisés. C'est formidable. En gros, l'ordinateur ne t'affiche que ce qui t'intéresse. Si tu aimes les grosses voitures, les idées de Robert Nozick et les clips de Britney Spears, tu n'as aucun risque de tomber sur des choses concernant les particules fines, Marx ou les suites pour violoncelle seul de Bach. Et réciproquement. Ça fait gagner un temps fou, ton attention n'est plus polluée par des infos sans intérêts. Accessoirement, j'observe que ça limite considérablement les risques de polémiques stériles entre des gens qui, de toute façon, ne sont pas faits pour s'entendre : grâce aux réseaux sociaux, on ne dialogue qu'avec des types qui pensent à peu près la même chose que nous.

Moi je trouve ça bien. Seulement, comme j’avais décidé de me désintéresser de la France pour couper le cordon ombilical, je n'en ai plus aucune nouvelle depuis un moment. Encore une fois je pourrais faire des recherches. Mais j'ai peur que les résultats soient un peu faussés par mes nouveaux centres d'intérêts.

Allez, écris-moi, quoi !

Je t'embrasse,

Paul 



\section{Adresse au peuple qui part sans en laisser, d'adresse}

Fragment d'une tablette retrouvée dans les Hautes Terres.

Traduit du dédalien par la rédaction

Dédaliennes, Dédaliens,

Nous voici réunis pour le grand départ. Ici, on fausse compagnie aux Utopistes!

Les histoires ne nous font pas peur. Il s'en est passé des choses ici, et nous ne manquons jamais d'entrain. Souvent nous allons même - on nous l'aura assez reproché - jusqu'à nous dépenser sans compter pour des causes perdues.

Mais cette histoire d'Utopie, non décidement, ne passera pas par nous. Qui sont ces visiteurs venus d'outre-espace, et qui sait, peut-être d'outre-temps, pour proclamer avec tant d'assurance que notre Dédalie serait leur soi-disant Utopie? De quelle éminente prescience prétendent-ils se prévaloir? Nous sommes las d'être pris pour les Utopistes que nous ne sommes pas.

Sans relâche nous les avons avertis : que nos us et coutumes ameutent vos méninges de philosophes et vos cabinets de curiosités, fort bien! Mais nous n'acceptons pas que vous en fassiez un folklore, et de nous des bêtes de foire. À vous en croire, il fut un temps où Utopie était le nom que nous donnions à notre pays. Même en admettant cette (pure) coïncidence, nous ne sommes pas la chimère démagogique que vous décrivez! Votre pays extraordinaire n'est pas de ce monde!

Vaines exhortations.

Aujourd'hui donc notre décision est prise. Puisqu'eux ne peuvent ni ne veulent nous laisser en paix, nous partirons. En fait d'Utopie, le désert.

Faussons compagnie aux Utopistes! 



\section{Institut für Dedalienforschung}

Fragment d'une tablette retrouvée dans les Hautes Terres.

Traduit de l'allemand par la rédaction

Objet : Rapport d'avancement de la mission Dédalie

Madame la présidente,

Conformément à notre convention d'excellence DAI-1957-DF-001, j’ai l'honneur de vous adresser ci-joint le rapport d'avancement concernant les mois 198 à 210 de la mission en zone 51, alias Dédalie.

Sont également inclus en annexe les tableaux budgétaires prévisionnels préparés par notre Institut de recherches dédaliennes en vue de la prochaine campagne d'attribution des subventions décennales.

À l'heure où l'Institut se dote d'un Kindergarten et d'une école primaire bilingue allemand-dédalien pour les enfants de ses résidents permanents, je ne saurais trop souligner, Madame la présidente, combien la survie culturelle de notre programme dépend de votre soutien.

Avec mes meilleurs respects,

Prof. em. Dr. rer. nat. Dr. phil. Ludwine Heimelig

Directrice déléguée

Institut de recherches dédaliennes

Zone 51 - Poste restante

[Encodage algorithmique indéchiffrable] 



\section{Cher François,}

Encore vu Georges. Il fait partie d'un groupuscule qui en a assez de cette société. Ils rédigent ensemble une sorte d'utopie, à paraître dans une revue confidentielle qu'ils seront sans doute les seuls à lire. Leur grande idée, c'est de faire «l'an $01 »$. Comme Gébé, oui. Ils ont d'ailleurs le même mot d'ordre que lui : «On arrête tout et on réfléchit». Entre nous, je leur souhaite bien du courage.

Bons baisers de Dédalie,

Paul 



\section{Nouvelle génération}

Fragment d'une tablette retrouvée dans les Hautes Terres.

Traduit du dédalien et annoté par la rédaction

Les Vieux sont partis. Anciens et matériel funéraire compris. Les porteurs étaient chargés à craquer. Ils sont partis pour ne plus revenir.

Nous avons fait mine d'acquiescer à cette histoire de retour au "pays ", à laquelle pourtant aucun d'entre nous ne prête sérieusement foi. La nostalgie a pu tarauder les Anciens, soit. Mais les Vieux, qui pour la plupart étaient ici depuis l'enfance? Nous autres sommes nés et avons grandi ici : vous ne nous ferez pas croire qu'on puisse détaler ainsi pour une choucroute fortiche ${ }^{1}$.

La vérité c'est que leur départ devait être planifié de longue date. La recolonisation de Dédalie était un programme expérimental, assorti d'un protocole générationnel rigoureux. Le principe en était que la continuité biologique ne puisse fonder aucune continuité politique. «Loin des Vieux, loin du cœur», comme ils disaient en éclatant d'un petit rire étouffé (ou d'un sanglot). Nous étions entre nous, laissés à nos dadas et à nos fantaisies, à nos nobles mensonges. On aurait dit que nous étions tous strictement autochtones : nés d'ici, d'une seule et même terre. Avec pour univers, la palette graphique du désert. Avez-vous déjà été au désert? Un paradis pour caméléons! C'est là comment nous avons été édifiés.

Les Vieux avaient donc prévu de s'effacer. Le budget devait être clos avec leur départ, voilà tout. C'était leur contrat originel à eux ${ }^{2}$. Des clauses trop anciennes, ou trop secrètes, pour que notre génération spontanée puisse en avoir connaissance. Ce serait d'ailleurs en vain qu'on nous demanderait sur quels registres cette charte de nos libertés a été consignée. Les Vieux tenaient des archives, paraît-il ; mais ils sont - cela

1. Cette occurrence laisse supposer qu'il s'agit là d'une probable déformation argotique dérivée de «Francfort» (NDT).

2. L'allusion au texte de David Hume «Of the Original Contract», dont ce paragraphe et le suivant sont au demeurant très inspirés, semble limpide. Mais en l'état actuel des connaissances des protocoles graphiques dédaliens, il n'est pas établi que l'auteur eût accès à la version originale du texte anglais ou à quelque traduction (NDT). 
va sans dire - partis avec. Hors d'ici les bilans d'étape, les rapports de synthèse et les projets décennaux ! L'essentiel est resté : la Graphothèque, qui est tout le contraire. Nulle chaîne de transmission ne l'entrave, nul syllogisme de funeste copiste. Lorsqu'ils ne sont pas au désert, nos petits démons s'égaillent sur tablette. Chacun de nous a bien dû vivre six mille ans du monde entier.

À quoi ressemble une société où l'ancienne génération s'efface subitement alors qu'au même moment la suivante fend la chrysalide? Nous serons si j'en crois mes sources (et elles sont pléthoriques) les premiers à pouvoir en faire l'expérience. Je ne connais guère en effet d'autre société où les Vieux se soient jusqu'au dernier si sciemment effacés - je veux dire, sans qu'aucune contingence historique ne l'exige. Et donc sans que les traces de leur passage n'exigent non plus le recueillement et la commémoration. L'histoire des populations avant nous ne manque pas de pyramides des âges éventrées. Mais une pyramide sans base ni sommet?

C'est là que les Vieux, il faut le reconnaître, ont été impériaux jusqu'au bout.

Nous voici entre nous : rien que des Dédaliens de souche! Une petite centaine de têtes. En compte calendaire fortiche, les aînés doivent avoir dans les douze ans. Les plus jeunes commencent à peine à lire. Nous y pourvoirons. Maintenant que le «pays» ne compte plus, à nous les coudées franches. Douze ans : je ne laisserai personne dire que ce ne soit pas le plus bel âge de la vie.

Déjà, nous allons changer de nom. Je ne sais d'où nous est venu celui de Dédalie - sans doute de quelques Anciens confits dans l'Académie, venus ici costumés en nouveaux Thésée. Désormais, trêve de blague. Nous nous sommes donné le mot:

- On dirait qu'ici ce serait l'Utopie.

Aussi, cela fait une paye que nous y avions réfléchi. Nous étions même tombés d'accord avant l'enlèvement des Vieux. Le secret était bien gardé. Une fois seulement l'un de nous se fit surprendre. En d'autres temps et lieux, cette gaffe nous aurait sans doute valu des châtiments corporels bien sentis.

On dit qu'il n'y a pas de progrès, mais simple déplacement des terrce incognitæ. Maintenant que nous voici livrés à nous-mêmes, nous allons être servis. Néanmoins notre religion est faite, et qu'on se le tienne pour dit :

- On dirait qu'il n'y aurait d'Utopie qu'ici. 


\section{Good morning, Mister Sborge...}

- Good morning, Mister Sborge, how are you?

- I'm fine, John, thank you.

- Would you mind signing this form for me please?

- Sure. Is RMC693 waiting for me?

- Absolutely. I am going to let It know that you arrived.

D'un geste vif, Walker Sborge enleva sa gabardine, puis s'enfonça, avec un soupir d'aise, dans un fauteuil moelleux aux armes de l'Association des Bibliophiles Chimériques de Dédalie. Quelle bonne idée, pensa-t-il, d'avoir acheté quelques dizaines de milliers d'hectares pour y installer le Rare and Manuscript Center, ce lieu de confusion totale entre la littérature et la vie. C'était l'aboutissement d'un plan concerté pendant des années par quelques amis amateurs de livres et de sensations fortes.

Les choses se passaient ainsi : ayant porté son choix sur une des «expériences» décrites dans un catalogue en ligne, le lecteur prenait rendez-vous en toute discrétion avec un conservateur d'apparence humaine, afin de préciser ses attentes. Concernant le «livre support», tout était passé soigneusement en revue : la langue, le format, la reliure, le papier, la typographie, et les illustrations. Luxe ultime : on se voyait offrir l'option de récrire le livre, en totalité ou en partie. Alors, Hector ou Geronimo gagnaient la guerre; Berl Pickett parvenait à réformer Livingston, Montana; Anna Karenine et Matthew Crowley ne mouraient plus, ou dans des circonstances moins absurdes; les œuvres complètes de Victor Hugo étaient expurgées de «La Vache», enfin il redevenait possible d'utiliser le titre La possibilité d'une île comme si Houellebecq n'avait jamais existé.

La deuxième étape était appelée «mise en condition», et s'effectuait dans une pièce imitée du salon de lecture de Kanazawa telle qu'il existait au xve siècle, ou inspirée de la bibliothèque du Nautilus. L'atmosphère musicale et olfactive, ainsi que les alcools et les drogues, étaient en rapport avec le livre support. Tout autour du sofa s'amoncelaient panoplies et accessoires piochés dans les collections de RMC : elles contenaient aussi bien l'armure de Don Quichotte que le pousse-pousse de Jiangxi.

À un degré d'immersion dans le texte équivalent à la perte de conscience claire (celle-ci mesurée par un capteur cérébral), le lecteur pouvait «poursuivre» ou «réaliser» la fiction ou le poème, sans presque aucune 
limitation dans le temps ni dans l'espace : ah, jouir d'une fellation dispensée par Calypso, ou par l'amant de Lady Chatterley; mmm, se régaler d'un tartare comme dans Michel Strogoff, de pirojki et de skorodumki comme dans Les Âmes mortes, ou de fraises écrasées dans de la crème comme dans la Recherche; woa, partir en promenade dans une carriole tirée par Cadichon, ou dans le ventre d'un monstre marin comme Jonas -- ou Pinocchio ; ô, se retrouver transporté dans les tranchées d'À l'Ouest, rien de nouveau, ou à Lilliput (comme disait l'un des amis de Sborge : «Qui n'a rêvé d'éteindre l'incendie d'un palais en pissant dessus ?») ; «à Mégara, faubourg de Carthage, dans les jardins d'Hamilcar», ou encore au pays de Tendre; et pour peu qu'ils suivissent leurs instincts les plus refoulés et ne regardassent pas à la dépense, les plus audacieux prenaient leur part d'un sacrifice humain chez les Mayas, ou d'une partouze sadienne, car le château de Silling avait été reconstruit spécialement pour l'occasion, à quelques centaines de kilomètres au nord.

Après? Eh bien, on se réveillait, deux heures ou douze ans plus tard, mais toujours avec une trace : une indigestion, une blessure mentale ou une infirmité physique (un bras en moins, un carcinome, des cheveux blancs); on éprouvait un sentiment de plénitude ou de culpabilité; une impression d'urgence («Ehe es einviertel acht schlägt muss ich unbedingt das Bett verlassen haben») ou de désespoir. En moins glorieux, c'était un petit creux après un jeûne de plusieurs mois, assis en tailleur sous un arbre malingre -- à moins que l'on n'ait attrapé un torticoli, à force d'admirer chaque gratte-ciel de New York avec Paul Morand pour guide. Certains ne revenaient jamais dans le monde dit réel, qui s'étaient trop vanté avant le grand sommeil d'hésiter entre la bouche d'un pistolet et les pieds de la Croix. Une clause de «non-réveil» était naturellement prévue dans le contrat.

$$
* * *
$$

Sborge sentit une délicate pression sur son bras, et ouvrit les yeux. RMC693 lui souriait. 


\section{Kit}

Campanella, Tommaso, La Cité du Soleil (éd. L. Firpo, trad. A. Tripet), Genève, Droz, 1972 [1602].

Flaubert, Gustave, Madame Bovary (édition B. Didier), Paris, LGE, 1983 [1856].

—, «Un cœur simple» in Trois contes (introduction et notes P.-M. de Biasi), Paris, LGE, 1999 [1877].

-, Bouvard et Pécuchet (chronologie et préface J. Suffel), Paris, GF Flammarion, 1966 [1879].

Foucault, Michel, Histoire de la sexualité, t. I, La volonté de savoir, Paris, Gallimard, 1976.

GoldFrapp, Felt Mountain, Mute, 2000.

Hume, David, 4 Essais politiques (éd. bilingue, trad. F. Grandjean et al.), Toulouse, Éditions Trans-Europ-Repress, 1981.

Le Bras, Hervé, Essai de géométrie sociale, Paris, Odile Jacob, 2000.

Mannheim, Karl, Le Problème des générations (trad. G. Mauger et N. Perivolaropoulou), Paris, Nathan, 1990 [1928].

MıLo, Daniel S., "Aspects de la survie culturelle », thèse de 3e cycle, Paris, École des hautes études en sciences sociales, 1985.

Strauss, Leo, Droit naturel et histoire (trad. M. Nathan et É. de Dampierre), Paris, Plon, 1954 [1953].

Van Vogt, A. E., Le Monde des Ā (trad. B. Vian), Paris, Hachette / Gallimard, 1953 [éd. orig. 1945].

Verbinski, Gore, Rango, Nickelodeon Movies / Blind Wink / GK Films, 2011. 